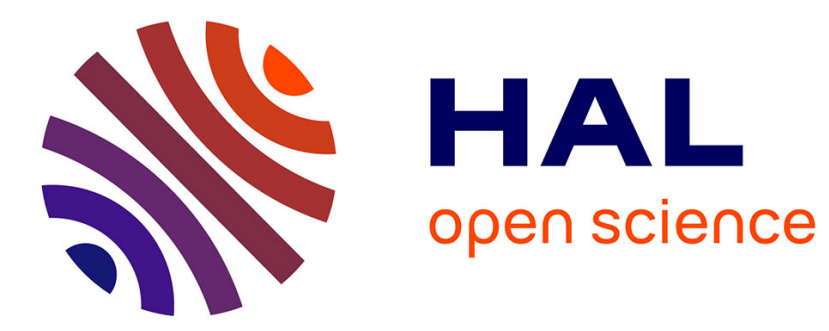

\title{
Thermal convection in three-dimensional fractured porous media
}

\author{
C. Mezon, V. Mourzenko, J.-F. Thovert, Raphaël Antoine, Fabrice J. \\ Fontaine, Anthony Finizola, P. Adler
}

\section{- To cite this version:}

C. Mezon, V. Mourzenko, J.-F. Thovert, Raphaël Antoine, Fabrice J. Fontaine, et al.. Thermal convection in three-dimensional fractured porous media. Physical Review E , 2018, 97 (1), pp.013106. 10.1103/PhysRevE.97.013106 . hal-03025954

\section{HAL Id: hal-03025954 \\ https://hal.science/hal-03025954}

Submitted on 2 Mar 2021

HAL is a multi-disciplinary open access archive for the deposit and dissemination of scientific research documents, whether they are published or not. The documents may come from teaching and research institutions in France or abroad, or from public or private research centers.
L'archive ouverte pluridisciplinaire HAL, est destinée au dépôt et à la diffusion de documents scientifiques de niveau recherche, publiés ou non, émanant des établissements d'enseignement et de recherche français ou étrangers, des laboratoires publics ou privés. 


\title{
Thermal convection in three-dimensional fractured porous media
}

\author{
C. Mezon, ${ }^{1,2,3,{ }^{*}}$ V. V. Mourzenko, ${ }^{4}$ J.-F. Thovert, ${ }^{4}$ R. Antoine,${ }^{2}$ F. Fontaine, ${ }^{3}$ A. Finizola, ${ }^{3}$ and P. M. Adler ${ }^{1}$ \\ ${ }^{1}$ UPMC METIS, Boîte 105, 4 Place Jussieu, 75252 Paris Cedex 05, France \\ ${ }^{2}$ CEREMA, Direction territoriale Normandie Centre, Laboratoire de Rouen, Groupe Sciences de la Terre, Le Grand Quevilly, France \\ ${ }^{3}$ Laboratoire GéoSciences Réunion, Université de la Réunion, Institut de Physique du Globe de Paris, \\ Sorbonne Paris Cité, CNRS UMR 7154, Saint Denis, La Réunion, France \\ ${ }^{4}$ Institut Pprime, CNRS, SP2MI, Boîte Postale 30179, 86962 Futuroscope Chasseneuil Cedex, France
}

(Received 4 May 2017; published 11 January 2018)

\begin{abstract}
Thermal convection is numerically computed in three-dimensional (3D) fluid saturated isotropically fractured porous media. Fractures are randomly inserted as two-dimensional (2D) convex polygons. Flow is governed by Darcy's 2D and 3D laws in the fractures and in the porous medium, respectively; exchanges take place between these two structures. Results for unfractured porous media are in agreement with known theoretical predictions. The influence of parameters such as the fracture aperture (or fracture transmissivity) and the fracture density on the heat released by the whole system is studied for Rayleigh numbers up to 150 in cubic boxes with closed-top conditions. Then, fractured media are compared to homogeneous porous media with the same macroscopic properties. Three major results could be derived from this study. The behavior of the system, in terms of heat release, is determined as a function of fracture density and fracture transmissivity. First, the increase in the output flux with fracture density is linear over the range of fracture density tested. Second, the increase in output flux as a function of fracture transmissivity shows the importance of percolation. Third, results show that the effective approach is not always valid, and that the mismatch between the full calculations and the effective medium approach depends on the fracture density in a crucial way.
\end{abstract}

DOI: 10.1103/PhysRevE.97.013106

\section{INTRODUCTION}

For many years, the simulation of fluid flow and heat transfer in natural media has attracted the attention of geoscientists and mathematicians. Moreover, the increasing threat of a worldwide energy crisis, which had begun in the 1970s, has stimulated research in renewable energies, such as geothermal energy. The process of thermal convection in a porous medium is the driving mechanism in many geophysical situations where heat is transferred to the earth's surface from an energy source deep below ground.

The first models assumed that the solid matrix is homogeneous with constant properties, nondeformable, and chemically inert with respect to the fluid. The density of the Newtonian fluid varies only with temperature; no heat sources or sinks exist in the fluid, and thermal radiation and viscous dissipation are negligible. Under these conditions, the velocity and temperature distribution are described by the mass conservation equation, the macroscopic Darcy law, and the heat transfer equation as detailed in Sec. II (see also Ref. [1]).

Homogeneous isotropic porous media have been theoretically and experimentally addressed since the 1940s. The first studied configurations were the closed box and the horizontally infinite slab of porous medium, uniformly heated from below. The onset of a convective movement in such systems was studied analytically in Refs. [2,3]. If the Rayleigh number is below a critical value $\mathrm{Ra}_{c}$, the heat transfer is purely conductive and it is characterized by the Nusselt number, $\mathrm{Nu}=1$. Hence,

\footnotetext{
*Corresponding author: cecile.mezon@upmc.fr
}

$\mathrm{Ra}_{c}$ represents the minimal value for which vertical fluid motion is observed in the porous medium. When $\mathrm{Ra}$ is larger than $\mathrm{Ra}_{c}$, convection appears. The theoretical values for the onset of convection are $\mathrm{Ra}_{c}=4 \pi^{2}$ in closed-top conditions and 27.10 in open-top conditions [3,4]. These theoretical analyses were followed by experimental studies (see, e.g., Refs. [5-7]); the first numerical solutions did not appear before the mid-1960s $[4,8]$.

The equations were solved by a finite difference scheme [9,10] for Ra ranging from $\mathrm{Ra}_{c}$ to 350 . The Galerkin technique was used to treat the steady-state two-dimensional problem for Rayleigh numbers as large as $10 \mathrm{Ra}_{c}$ in Ref. [11]. It has been shown that when $\mathrm{Ra}$ increases, the system follows a series of transitions between two-dimensional (2D) convection patterns, from steady structures, to periodic patterns, to quasiperiodic ones, and, finally, to chaotic behavior. If $\mathrm{Ra}$ is small, there are always stable convective patterns, and when $\mathrm{Ra}>380$, stable unicellular regimes do not exist anymore [11]. But steady-state pluricellular convection can be stable for $\mathrm{Ra}>380$. The convection regime is stabilized by a reduction of the lateral extension of the convection cells [10]. The Galerkin approach is applied in Ref. [12] in order to study the stability of multicellular convection patterns in square domains; they found that one-cell regimes are stable up to $\mathrm{Ra} \approx 300-320$, that two-cell solutions become unstable for $\mathrm{Ra} \approx 650-700$, and that three-cell flows lose their stability when $\mathrm{Ra}>800-1000$. The evolution of the Nusselt number as a function of the Rayleigh number and of the aspect ratio of the convection cell in a horizontal porous slab is detailed in Ref. [10]. 
However, heterogeneities are always present in natural media. As a matter of fact, most geothermal reservoirs are fractured, and the fluid, even if stored in the porous matrix, tends to flow through the easiest paths, i.e., the fractures [13]. The problem of the representation of fractured reservoirs has received considerable attention.

The first approach is the double-porosity model introduced in Refs. [14,15], where two media overlap with exchanges between them. This simplified approach has been followed by many authors (e.g., [16-18]).

Another technique to include fractures in the medium is to consider areas of higher permeability than the embedding homogeneous media. Many of these contributions were reviewed in Ref. [19]. Among the late contributions using this technique, one can cite Refs. [20-24]; some of these codes are still 2D and use commercial software to discretize and solve the equations. They are specific since they model a given site and study its properties.

More recently, three-dimensional (3D) descriptions of porous media containing discrete planar fractures were proposed. Two subhorizontal geological layers including two subvertical faults that cross both layers were simulated in Ref. [25]; the flow and the heat transfer were calculated for a horizontal temperature gradient, considering four different permeabilities (two for the faults and two for the geological layers). The model of Ref. [26] was generated using the commercial software PETREL (Schlumberger) and represents the Molasse Basin, southern Bavaria, Germany. Numerical simulations of threedimensional media were undertaken in Ref. [27] to quantify the effects of transmissivity of a single fault embedded in a permeable matrix, in terms of fluid flow and heat transfer. To carry out numerical simulations of coupled fluid and heat transfer, the 3D mesh is imported in OpenGeoSys, which is a finite-element-based numerical simulator for coupled thermal, hydraulic, mechanical, and chemical (THMC) processes for both fractured and nonfractured porous media [28]. They showed that the permeability contrast between the fault and the matrix should be strong $\left(>10^{2}\right)$ for the faults to influence the pressure and temperature fields.

The present work is based on a three-dimensional discrete description of the fracture network and of the embedding matrix (cf. Ref. [29] for an overview); therefore, it belongs to the last class of models just mentioned. Isotropic fracture networks are modeled, and the matrix is permeable. Another important difference from the previous studies is that the resulting code is not applied to a particular field. The first objective of this paper is to provide quantitative information about the influence of the network parameters such as the dimensionless fracture transmissivity and the dimensionless fracture density on the heat released by the whole system. The second objective is to assess the validity of a homogeneous approach.

In Sec. II, the main definitions and the governing equations are provided. In Sec. III, the code is validated by benchmarks made for homogeneous porous media. In Sec. IV, the influence of the network parameters is studied; the effective permeability is calculated for each network, as previously proposed in Ref. [30]; and the results in fractured media and porous media with the same macroscopic properties are compared. The validity of the homogeneous approach is discussed in Sec. V.

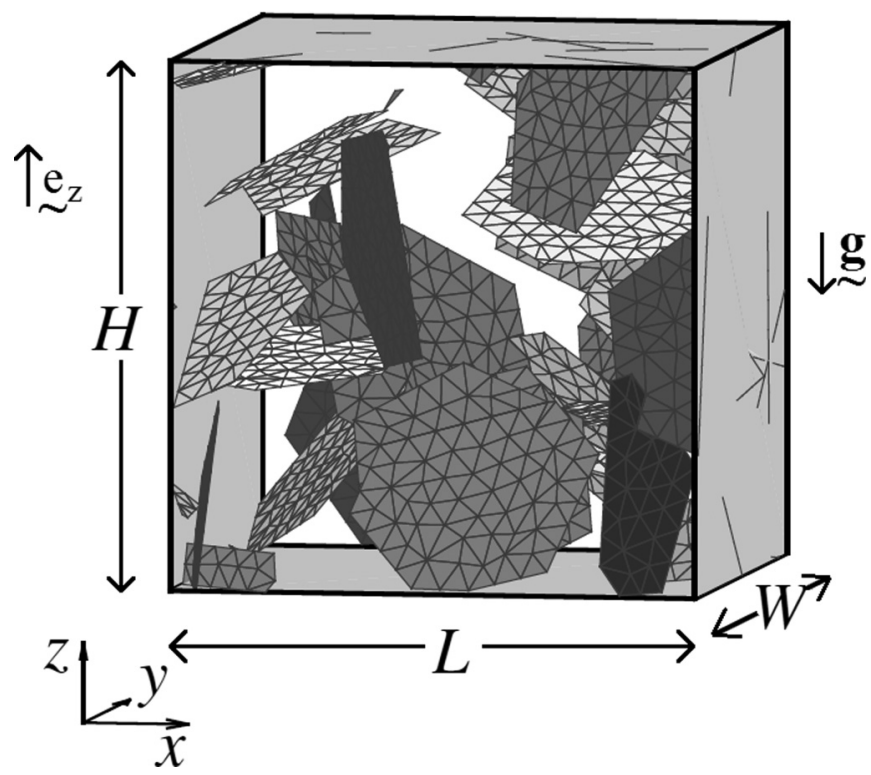

FIG. 1. An isotropic 3D fracture network composed of hexagonal fractures. The colors are only used to distinguish the various hexagons.

Finally, some concluding remarks end this paper and some extensions to this work are proposed.

\section{GENERAL}

\section{A. Theoretical framework}

In the present work, convective heat transfer in fractured porous media is addressed by direct numerical simulations of discrete fracture models, as illustrated in Fig. 1. The porous matrix is regarded as a continuous material of permeability $K_{m}$. Fractures are schematized as polygons inscribed in disks of radius $R$; their aperture is constant and equal to $b$, and the flow in these fractures is described by a two-dimensional form of Darcy's law. This section provides the governing equations.

\section{Flow equations}

The following equations are written in a quasisteady approximation, whereby it is assumed that the (very slow) variations of the medium properties and those of the fluid can be neglected. Since for liquids and gases the thermal expansion coefficient ranges between $10^{-3}$ and $10^{-4} \mathrm{~K}^{-1}$, and thus is very small, the Boussinesq approximation is usually introduced (see Ref. [10], for example), which states that the variations in the fluid density $\rho_{F}$ are negligible except in the body force term; it is valid when density changes remain small in comparison to the reference density $\rho_{F 0}$. No phase change occurs and the medium is fully saturated by the fluid.

Flow is governed by the continuity equation supplemented by Darcy's law,

$$
\begin{aligned}
\nabla \cdot \overline{\mathbf{v}} & =0 \\
\overline{\mathbf{v}} & =-\frac{K_{m}}{\mu}\left(\nabla P-\rho_{F} \mathbf{g}\right),
\end{aligned}
$$


where $P$ is the pressure, $\overline{\mathbf{v}}$ the mean seepage velocity, $\mu$ the viscosity, and $\mathbf{g}$ the gravity acceleration.

Similar equations are valid in the fractures [30,31]:

$$
\begin{aligned}
\nabla_{S} \cdot \mathbf{q}+[\overline{\mathbf{v}}] \cdot \mathbf{n} & =0, \\
\mathbf{q} & =-\frac{1}{\mu} \sigma_{f}\left[\nabla_{S} P-\rho_{F} \mathbf{g} \cdot(\mathbf{I}-\mathbf{n n})\right],
\end{aligned}
$$

where Eq. (2a) is a continuity equation including fluid exchanges with the embedding matrix and Eq. (2b) the twodimensional form of Darcy's law. $\mathbf{I}$ is the unit tensor. Here, $\boldsymbol{\nabla}_{S}$ denotes the two-dimensional gradient operator in the fracture plane, $\sigma_{f}$ the fracture transmissivity, $\mathbf{q}$ the flow rate per unit width (integral of flow velocity over the fracture aperture), $\mathbf{n}$ the unit normal vector to the fracture, and $[x]$ the difference between a quantity $x$ evaluated on the $+\mathbf{n}$ and $-\mathbf{n}$ sides of the fracture. Fractures are assumed to be open channels that offer no resistance to cross flows, and pressure is regarded as constant across the fracture aperture, and equal to the pressure in the matrix on both sides. This usual assumption does not imply that there is no flow between the fracture and the matrix since there generally exist pressure gradients on both sides of the fracture.

\section{Energy equations}

Local equilibrium is assumed everywhere, which means that in any volume element the fluid and the solid are at the same temperature $T$. In typical conditions, with physical parameters such as those in Table I and submillimeter pore sizes and fracture apertures, the characteristic time scale for local temperature equalization is at most a few seconds, i.e., quasi-instantaneous compared to all other processes.

When the compression work is neglected, the energy balance equation in the matrix reads [32]

$$
\begin{aligned}
& \frac{\partial}{\partial t}\left[\varepsilon \rho_{F} h_{F}^{*}+(1-\varepsilon) \rho_{S} h_{S}^{*}\right]+\nabla \cdot\left(\overline{\mathbf{v}} \rho_{F} h_{F}^{*}\right)-\nabla \cdot\left(\lambda_{m} \nabla T\right) \\
& \quad=0,
\end{aligned}
$$

where $\varepsilon$ is the medium porosity, $\lambda_{m}$ the effective conductivity of the porous medium, $\rho_{S}$ the solid matrix density, and $\left(h_{F}^{*}\right.$, $h_{S}^{*}$ ) the specific enthalpies of the liquid and solid phases.

Fractures are assumed to have no thermal resistance to normal temperature gradients, and their temperature is considered constant across the fracture aperture and equal to $T$ in the matrix on both sides. When the compression work is neglected, the energy balance reads $[33,34]$

$$
\begin{aligned}
\frac{\partial\left(b \rho_{F} h_{F}^{*}\right)}{\partial t} & +\nabla_{S} \cdot\left(\mathbf{q} \rho_{F} h_{F}^{*}\right)-\nabla_{S} \cdot\left(\Lambda \nabla_{S} T\right) \\
& +\left[\overline{\mathbf{v}} \rho_{F} h_{F}^{*}-\lambda_{m} \nabla T\right] \cdot \mathbf{n}=0,
\end{aligned}
$$

where $b$ is recalled to be the fracture aperture. Again the last term corresponds to the exchanges with the surrounding matrix.

\section{Constitutive equations}

The fluid- and solid-phase enthalpies and the fluid density are linear functions of the temperature:

$$
h_{F}^{*}=c_{F} T, \quad h_{S}^{*}=c_{S} T, \quad \rho_{F}=\rho_{F 0}\left[1-\alpha_{F}\left(T-T_{0}\right)\right],
$$

where $c_{S}$ and $c_{F}$ are the specific heat capacities of the solid phase and of the fluid phase, respectively; $\alpha_{F}$ is the coefficient of thermal volume expansion of the fluid; and $T_{0}$ is the corresponding reference value.

The effective thermal conductivity $\lambda_{m}$ of the porous medium is approximated by the elementary mixture rule [1]

$$
\lambda_{m}=\varepsilon \lambda_{F}+(1-\varepsilon) \lambda_{S},
$$

where $\lambda_{F}$ and $\lambda_{S}$ are the thermal conductivities of the fluid and solid phases, respectively.

The fractures are schematized as plane channels with an equivalent aperture $b$. Therefore, the fracture thermal transmissivity $\Lambda$ and the fracture hydraulic transmissivity $\sigma_{f}$ are given by

$$
\Lambda=b \lambda_{F}, \quad \sigma_{f}=\frac{b^{3}}{12}
$$

\section{Boundary and initial conditions}

Consider a bounded three-dimensional volume $L \times W \times H$ of fractured porous medium heated from below (Fig. 1). The vertical boundaries of dimensions $W \times H$ and $L \times H$ are assumed adiabatic and impermeable:

$$
\begin{aligned}
& x=0, L: \quad \bar{v}_{x}=0, q_{x}=0 ; \quad j_{x}=0, J_{x}=0, \\
& y=0, W: \quad \bar{v}_{y}=0, q_{y}=0 ; \quad j_{y}=0, J_{y}=0 .
\end{aligned}
$$

The bottom of the box, $z=0$, is always impermeable while the top, $z=H$, could be impermeable or permeable. In opentop cases, a constant pressure $P_{0}$ is imposed, while in closedtop cases a no-flow boundary condition is used. Open-top and closed-top problems are investigated in homogeneous porous media; in fractured porous media, only closed-top conditions are tested. A constant temperature $T_{0}$ is imposed at the top of the box; the temperature at the bottom is fixed at $T_{0}+\Delta T$. Therefore,

$$
\begin{aligned}
& z=0: \quad \bar{v}_{z}=0, \quad q_{z}=0 ; \quad T=T_{0}+\Delta T, \\
& z=H: \quad \bar{v}_{z}=0, \quad q_{z}=0, \quad \text { or } P=P_{0} ; \quad T=T_{0} .
\end{aligned}
$$

The situations of a thermally insulating top boundary and of transient stages are not considered here.

In most cases, simulations start from a homogeneous temperature field and an immobile fluid:

$$
t=0: \overline{\mathbf{v}}=0, \mathbf{q}=0, T=T_{0} .
$$

However, other initial conditions can be used in order to induce a specific flow pattern (see Sec. III B).

\section{Dimensionless formulation}

In order to reformulate the problem in dimensionless variables, consider the reference state, with uniform conduction from the bottom to the top of the system without fractures when the fluid is immobile. This elementary solution denoted 
TABLE I. Physical parameters for the simulations in a homogeneous porous medium. The value of $\Delta T$ corresponds to Ra $=50$ in the reference case.

\begin{tabular}{|c|c|c|}
\hline Density of the porous material & $\rho_{S}$ & $3 \times 10^{3} \mathrm{~kg} \mathrm{~m}^{-3}$ \\
\hline Density of the fluid & $\rho_{F 0}$ & $1 \times 10^{3} \mathrm{~kg} \mathrm{~m}^{-3}$ \\
\hline Thermal conductivity of the fluid & $\lambda_{F}$ & $0.6 \mathrm{~W} \mathrm{~m}^{-1} \mathrm{~K}^{-1}$ \\
\hline Thermal conductivity of the porous material & $\lambda_{S}$ & $3.5 \mathrm{~W} \mathrm{~m}^{-1} \mathrm{~K}^{-1}$ \\
\hline \multicolumn{3}{|c|}{ Effective thermal conductivity of the porous medium, } \\
\hline$\epsilon \lambda_{F}+(1-\epsilon) \lambda_{S}$ & $\lambda_{m}$ & $2.6 \mathrm{~W} \mathrm{~m}^{-1} \mathrm{~K}^{-1}$ \\
\hline Porosity of the porous material & $\varepsilon$ & 0.3 \\
\hline Permeability of the porous material & $K_{m}$ & $2 \times 10^{-11} \mathrm{~m}^{2}$ \\
\hline Temperature difference & $\Delta T$ & $76.2 \mathrm{~K}$ \\
\hline Viscosity of the fluid & $\mu_{0}$ & $10^{-3} \mathrm{~Pa} \mathrm{~s}$ \\
\hline Specific heat of the porous material & $c_{S}$ & $0.9 \times 10^{3} \mathrm{~J} \mathrm{~kg}^{-1} \mathrm{~K}^{-1}$ \\
\hline Specific heat of the fluid & $c_{F}$ & $4.2 \times 10^{3} \mathrm{~J} \mathrm{~kg}^{-1} \mathrm{~K}^{-1}$ \\
\hline Thermal expansion coefficient & $\alpha_{F}$ & $2.07 \times 10^{-4} \mathrm{~K}^{-1}$ \\
\hline Gravitational constant & $g$ & $9.81 \mathrm{~m} \mathrm{~s}^{-2}$ \\
\hline Box size & $H$ & $10 \mathrm{~m}$ \\
\hline
\end{tabular}

by the subscript $r$ can be written as

$$
\begin{aligned}
& \overline{\mathbf{v}}_{r}=0, \quad \rho_{F}=\rho_{F 0}\left[1-\alpha_{F} \Delta T\left(1-\frac{z}{H}\right)\right], \\
& T_{r}=T_{0}+\Delta T\left(1-\frac{z}{H}\right), \\
& P_{r}=P_{0}+\rho_{F 0} g(H-z)\left[1-\frac{1}{2} \alpha_{F} \Delta T\left(1-\frac{z}{H}\right)\right] .
\end{aligned}
$$

Except for the variations of $\rho_{F}$ in Eqs. (1b) and (2b), all the material and transport properties of the fluid and of the porous matrix are considered constant. We introduce the dimensionless variables

$$
x=H x^{\prime}, \quad \nabla=\frac{1}{H} \nabla^{\prime}, \quad t=\frac{\left[\epsilon \rho_{F 0} c_{F}+(1-\epsilon) \rho_{S} c_{S}\right] H^{2}}{\lambda_{m}} t^{\prime}
$$$$
\overline{\mathbf{v}}=\frac{\lambda_{m}}{\rho_{F 0} c_{F} H} \overline{\mathbf{v}}^{\prime}, \quad \mu=\mu_{0} \mu^{\prime},
$$

$T=T_{r}+\Delta T T^{\prime}, \quad P=P_{r}+\frac{\mu_{0} \lambda_{m}}{\rho_{F 0} c_{F} K_{m}} P^{\prime}$.

Equations for the matrix, Eqs. (1a), (1b), and (3), can be rewritten in dimensionless form as

$$
\begin{aligned}
\nabla^{\prime} \cdot \overline{\mathbf{v}}^{\prime}=0, \quad \overline{\mathbf{v}}^{\prime} & =-\nabla^{\prime} P^{\prime}+\operatorname{Ra} T^{\prime} \mathbf{e}_{z}, \\
\frac{\partial T^{\prime}}{\partial t^{\prime}}-v_{z}^{\prime}+\nabla^{\prime} \cdot\left(\overline{\mathbf{v}}^{\prime} T^{\prime}\right) & =\nabla^{\prime 2} T^{\prime},
\end{aligned}
$$

where $\mathbf{e}_{z}$ is the unit vector along the $z$ direction and oriented upwards (see Fig. 1) and $\mu^{\prime}=1$. The Rayleigh number Ra, which compares the buoyancy forces to the viscous forces, is defined as

$$
\mathrm{Ra}=\frac{\alpha_{F} \rho_{F 0}^{2} c_{F} g \Delta T K_{m} H}{\mu_{0} \lambda_{m}}
$$

The dimensionless fracture equations derived from Eqs. (2a), (2b), and (4) can be written as

$$
\begin{aligned}
& \nabla_{S}^{\prime} \cdot \mathbf{q}^{\prime}+\frac{1}{R^{\prime}}\left[\overline{\mathbf{v}}^{\prime}\right] \cdot \mathbf{n}=0 \\
& \mathbf{q}^{\prime}=\sigma_{f}^{\prime}\left[-\nabla_{S}^{\prime} P^{\prime}+\operatorname{Ra} T^{\prime}\left(\mathbf{e}_{z}-n_{z} \mathbf{n}\right)\right] \\
& \omega^{\prime} \frac{\partial T^{\prime}}{\partial t^{\prime}}-q_{z}^{\prime}\left(1-n_{z}^{2}\right)^{1 / 2}+\nabla_{S}^{\prime} \cdot\left(\mathbf{q}^{\prime} T^{\prime}\right)-\Lambda^{\prime} \nabla_{S}^{\prime 2} T^{\prime} \\
& \quad+\frac{1}{R^{\prime}}\left[\overline{\mathbf{v}}^{\prime} T^{\prime}-\nabla^{\prime} T^{\prime}\right] \cdot \mathbf{n}=0
\end{aligned}
$$

with the following dimensionless parameters:

$$
\begin{aligned}
\sigma_{f}^{\prime} & =\frac{\sigma_{f}}{K_{m} R}, \quad \omega^{\prime}=\frac{b \rho_{F 0} c_{F}}{R\left[\epsilon \rho_{F 0} c_{F}+(1-\epsilon) \rho_{S} c_{S}\right]}, \\
\Lambda^{\prime} & =\frac{\Lambda}{\lambda_{m} R}, \quad R^{\prime}=\frac{R}{H},
\end{aligned}
$$

together with the relation

$$
\mathbf{q}=\frac{\lambda_{m} R}{\rho_{F 0} c_{F} H} \mathbf{q}^{\prime} .
$$

Boundary conditions (8) and (9) become

$$
\begin{aligned}
& x^{\prime}=0, L^{\prime}: \quad \bar{v}_{x}^{\prime}=0, \quad q_{x}^{\prime}=0 ; \quad j_{x}^{\prime}=0, \quad J_{x}^{\prime}=0, \\
& y^{\prime}=0, W^{\prime}: \quad \bar{v}_{y}^{\prime}=0, \quad q_{y}^{\prime}=0 ; \quad j_{y}^{\prime}=0, \quad J_{y}^{\prime}=0 \\
& z^{\prime}=0: \quad \bar{v}_{z}^{\prime}=0, \quad q_{z}^{\prime}=0 ; \quad T^{\prime}=1, \\
& z^{\prime}=1: \quad \bar{v}_{z}^{\prime}=0, \quad q_{z}^{\prime}=0, \quad \text { or } \quad P^{\prime}=0 ; \quad T^{\prime}=0
\end{aligned}
$$

where $L^{\prime}=L / H$ and $W^{\prime}=W / H$.

In order to characterize the heat transfer through the system, the Nusselt number is introduced. The heat flux through the bottom surface $z=0$ is

$$
Q_{T}=\left.\int_{0}^{L} d x \int_{0}^{W} d y j_{z}\right|_{z=0}+\left.\sum_{f r \cap\{z=0\}} \int_{f r} d l J_{z}\right|_{z=0},
$$

where the last term is the sum over all intersections of the bottom plane $z=0$ with fractures; the integral runs over the 
whole length of each intersection line. According to Eqs. (9), $Q_{T}$ can be written as

$$
Q_{T}=-\left.\lambda_{m} \int_{0}^{L} d x \int_{0}^{W} d y \frac{\partial T}{\partial z}\right|_{z=0}-\left.\Lambda \sum_{f r \cap z=0} \int_{f r} d l \frac{\partial T_{f}}{\partial z}\right|_{z=0} .
$$

For pure conduction without any fracture [Eq. (11)], the heat flux $Q_{T}$ is

$$
Q_{T r}=\lambda_{m} \frac{\Delta T}{H} W L .
$$

The Nusselt number is defined as the ratio between $Q_{T}$ and $Q_{T r}$ :

$$
\mathrm{Nu}=\frac{Q_{T} H}{\lambda_{m} W L \Delta T} .
$$

$\mathrm{Nu}$ compares the real heat transfer to the one which would be produced by pure conduction.

\section{B. Numerical solution}

The numerical solution consists of three major steps, namely, meshing, discretization, and resolution by conjugate gradients algorithms.

All the computations for fractured media are conducted in cubic domains $(L=W=H)$. Two-dimensional square domains $(L=H)$ are also considered for homogeneous media in Sec. III A, for comparison purposes. The same simulation tool is used, with a very small $W$.

\section{Fracture generation and meshing}

Fractures are inserted in the porous matrix according to the procedure detailed by Ref. [35]. The fractures are modeled as plane polygons inscribed in circles of radius $R$. The centers of these polygons are uniformly distributed in space, and their normals are isotropically distributed; fractures are hexagons of a given size and characterized by a unique fracture aperture $b$. They are characterized by the network density $\rho$, equal to the number of fracture centers per unit volume. $\rho^{\prime}$ can be expressed in a dimensionless form:

$$
\rho^{\prime}=\rho V_{\mathrm{ex}}, \quad \text { with } \quad V_{\mathrm{ex}}=\frac{1}{2} A P,
$$

where $A$ is the fracture area, $P$ its perimeter, and $V_{\text {ex }}$ the excluded volume. The dimensionless density $\rho^{\prime}$ is also equal to the average number of intersections per fracture for isotropic networks. References $[35,36]$ have shown that $\rho^{\prime}$ controls most of the topological and hydraulic properties of the network.

Since the fractures intersect randomly, the most natural discretization method is an unstructured triangulation, with a maximal discretization length $\delta_{M}$ (see Ref. [36]). Each fracture is tesselated by triangles. Triangulation starts from fracture edges and fractures intersections, and fractures are triangulated according to an advancing front technique; i.e., fracture contours and fracture intersections are discretized first and progressively the rest of the fracture surface is triangulated, adding a third point to an existing segment, at a distance $\delta$ not exceeding a predefined discretization length $\delta_{M}$ (see Ref. [36]).
When the fracture network is totally triangulated, the space between the fractures is meshed by tetrahedra with the same maximal length $\delta_{M}$.

\section{Finite volume formulation}

The finite volume method is used in order to discretize the flow and energy balance equations (1a)-(4). The unknowns (pressure and temperature) are determined at the nodes of the tetrahedral mesh. The control volume $\Omega_{i}$ around the node $i$ is simply one-fourth of the volume of each incident tetrahedron to this node. Similarly to Ref. [37], nodes in fractures are considered centers of common control volumes for the fracture and for a neighbor portion of the porous matrix; hence, an explicit evaluation of exchange terms in Eq. (16b) is not necessary.

The linearized Darcy equation yields a linear system of equations for pressure that is solved by an ordinary conjugate gradient method. The velocity $\overline{\mathbf{v}}$ and $\mathbf{q}$ are subsequently derived by using Eqs. (1b) and (2b) from the pressure field. The same methodology is applied to the energy equation.

Since a quasisteady approximation is applied, $\rho_{F}$ is regarded as constant during a time step and equal to the value at the beginning of the time step. The same applies for the flow velocities which appear in the energy balance equation. Since flow is modified only as a consequence of the changes in the material properties, its variations are ignored during the solution of the latter. The flow problem is solved at the beginning of the time step. The energy balance equations are solved next. A time-implicit, first-order discretization is applied. If the convective transport is described by a simple upwind model, the problem can be solved directly by a biconjugate gradient method. However, this method generates numerical diffusion. A variant of the flux-corrected method proposed in Ref. [38] for multidimensional transport problems on unstructured grids is used in this work. When the flux limiting scheme is used, the problem is nonlinear and it has to be solved iteratively.

\section{The dimensionless parameters}

It might be useful at the end of this section to provide a complete list of dimensionless parameters. In principle, any quantity such as the Nusselt number is a function of these parameters. For the sake of clarity, they can be divided into several classes:

overall geometrical parameters $L^{\prime}, W^{\prime}$,

overall thermal parameter $\mathrm{Ra}$,

parameters characterizing the fractures $\rho^{\prime}, R^{\prime}, \sigma_{f}^{\prime}, \Lambda^{\prime}, \omega^{\prime}$,

numerical parameters $\delta_{M}^{\prime}=\delta_{M} / H$.

The realistic ranges for parameters $(23 \mathrm{c})$, for fractures a few meters in size with apertures $\sim 0.1$ to $\sim 1 \mathrm{~mm}$, can be deduced from the typical values of the fluid, solid, and porous matrix coefficients given in Table I. The hydraulic transmissivity $\sigma_{f}^{\prime}$ of the fractures ranges from $\sim 10^{-1}$ to $\sim 10$. Their thermal transmissivity is very small $\left(\Lambda^{\prime}<10^{-3}\right)$, as 
well as their thermal inertia compared to that of the matrix $\left(\omega^{\prime}<10^{-3}\right)$. Fracture networks with density $\rho^{\prime}=0.5-10$, ranging respectively far below and far above the percolation threshold, are considered with constant geometrical parameters $L^{\prime}=W^{\prime}=1$ and $R^{\prime}=0.2$, and Rayleigh numbers up to 300 (homogeneous media) or 150 (fractured media). Finally, the discretization parameter $\delta_{M}^{\prime}$ is set small enough to eliminate most of its influence on the result.

Note that given the fluid, solid, and porous matrix properties, $\sigma_{f}^{\prime}, \Lambda^{\prime}$, and $\omega^{\prime}$ are not independent if the fractures are plane open channels, since they result from only two additional parameters, namely, $b$ and $R$. However, they can change independently if other hypotheses are made for the fractures, such as rough walls or partial filling, or if the material properties are modified. Nevertheless, $\Lambda^{\prime} \ll 1$ and $\omega^{\prime} \ll 1$ are expected to apply in any realistic situation.

\section{THERMAL CONVECTION IN HOMOGENEOUS POROUS MEDIA}

To the main features of thermal convection in a homogeneous porous medium which were briefly recalled in Sec. II, a few remarks on 2D and 3D convection should be added. In 3D confined systems, stable convective regimes are observed for $4 \pi^{2}<\mathrm{Ra}<240-300$; only transient regimes exist above these values [1]. 3D convection in cubic boxes is studied numerically in Ref. [39], where it was observed that 2D convection flows have larger $\mathrm{Nu}$ than $3 \mathrm{D}$ convection flows for $\mathrm{Ra} \leqslant 97$ and that two-dimensional cells can develop as well as $3 \mathrm{D}$ steady patterns, depending on the initial conditions without any particular physical preference for either one. A sequence of transitions between flow regimes is discussed in Ref. [40]: at $\mathrm{Ra}=550$, the system evolves from a symmetric steady convection regime to a nonsymmetric one; for $\mathrm{Ra}>575$, the flow becomes oscillatory with a single frequency; then the flow becomes quasiperiodic for Ra larger than 650-680; up to $\mathrm{Ra} \sim 725$ a single frequency exists; and for $\mathrm{Ra}>725$ the regime becomes again quasiperiodic. The interested reader could also refer to Ref. [1] and references therein for more information.

This section is divided into two parts. The first part is a detailed analysis of the establishment of convection in a thin square vertical slab of porous medium. In the second part, results for stable steady-state two-dimensional and threedimensional convection in a cubic box are benchmarked for Ra up to 300 .

\section{A. Reference case}

One of the simplest situations consists of a homogeneous porous medium fully saturated by a single fluid. Vertical walls are impermeable and adiabatic. Horizontal walls are impermeable and their temperature is kept constant over the simulation. The simulation is started with a uniform temperature in the medium, equal to the temperature at the top of the box.

An essentially two-dimensional reference case $\left(L^{\prime}=1\right.$, $\left.W^{\prime}=0.025\right)$ with $\mathrm{Ra}=50$ is examined in detail. For illustration, a set of representative physical parameters that correspond to this situation is provided in Table I.
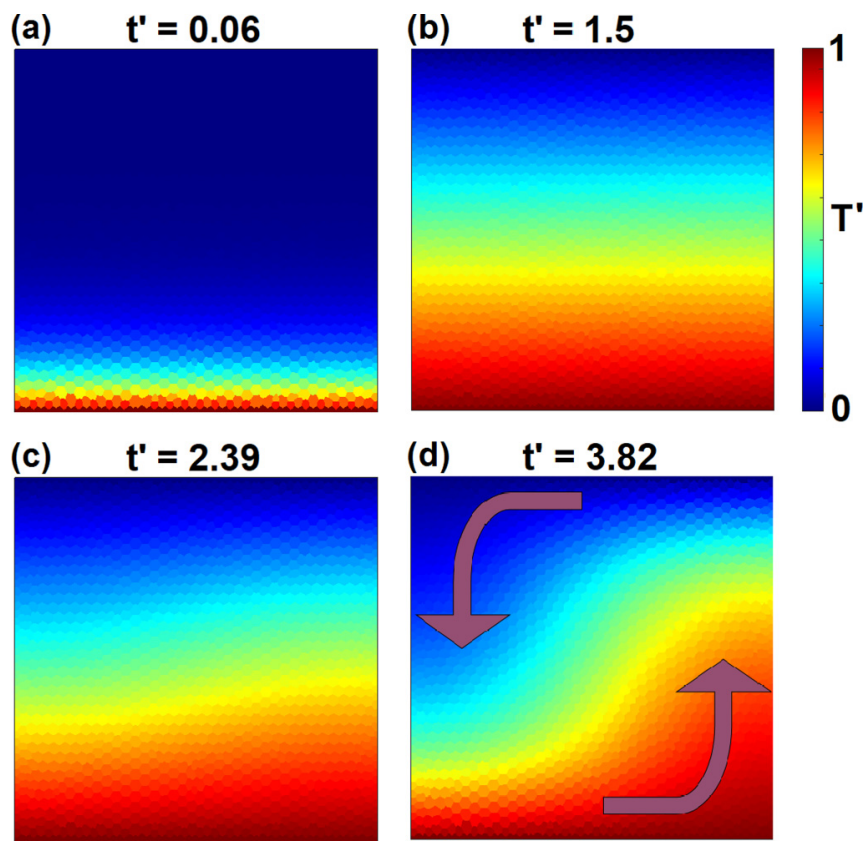

FIG. 2. Evolution of the temperature field in a vertical cross section of a pseudo-two-dimensional box $(1 \times 1 \times 0.025)$. $\mathrm{Ra}=50$, $\delta_{M}^{\prime}=0.0167$. (a) The simulation starts from a uniform temperature field. (b) A conductive temperature field is established. (c) Because of the overcritical $\mathrm{Ra}$, the conductive temperature field is destabilized. (d) Steady-state $2 \mathrm{D}$ convection for $\mathrm{Ra}=50$.

Unstructured tetrahedral grids with discretizations $\delta_{M}^{\prime}=$ $0.05,0.033,0.025$, and 0.017 are used for the numerical solutions. The time step $t_{\text {step }}^{\prime}$ is set accordingly, so that the temperature field does not change significantly over one time increment. Diffusive and advective characteristic time scales are associated with the grid resolution, namely, $\tau_{\text {cond }}^{\prime}=\delta_{M}^{\prime 2}$ and $\tau_{\mathrm{adv}}^{\prime}=\delta_{M}^{\prime} / v_{\max }^{\prime}$, where $v_{\max }^{\prime}$ is the largest velocity observed in the system. The time step is set to the smallest of these two values, which in practice is always $\tau_{\text {cond }}^{\prime}$.

Figure 2 shows the evolution of the temperature field in a two-dimensional box. $\mathrm{Ra}=50$ is slightly larger than the critical value $4 \pi^{2}$ which explains the establishment of a conductive pattern of temperature distribution in a first stage [Fig. 2(b)]. Then, the conductive temperature field is destabilized [Fig. 2(c)]. Finally, the system reaches a convective steady state [Fig. 2(d)]. The cellular motion of the fluid is indicated by the arrows displayed in Fig. 2(d).

Figure 3 represents the evolution of $\mathrm{Nu}$ for three different grid sizes $\delta_{M}^{\prime}=0.033,0.05$, and 0.017. As seen in Fig. 2, the first step is the establishment of a conductive temperature field which is subsequently destabilized; finally, the steadystate temperature field is reached. Hence, the evolution of $\mathrm{Nu}$ follows the same steps. As long as $\mathrm{Nu}<1$, conduction controls the establishment of the temperature field. This field is destabilized when $\mathrm{Nu}>1$, and the steady-state temperature field is established when $\mathrm{Nu}$ is constant with time. During the simulations, the destabilization was observed to occur at different times depending on the grid resolution (see Fig. 3).

The system is supposed to reach steady state when the output flux at the bottom and at the top of the box are equal (within five significant digits), after the onset of convection. Then, the 


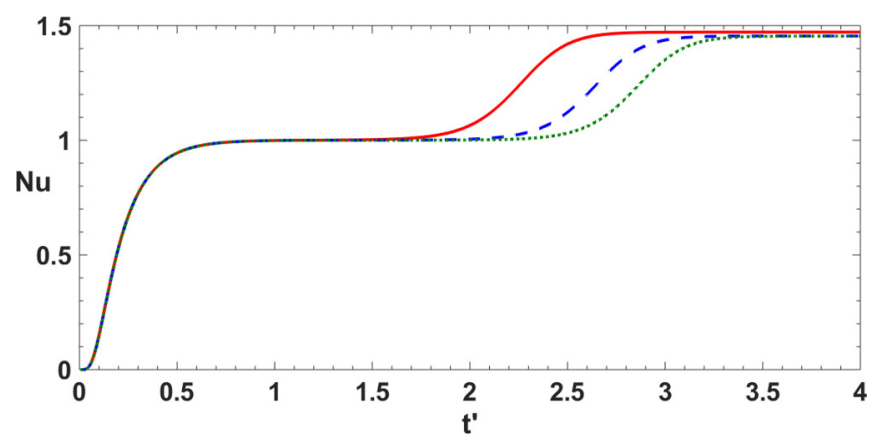

FIG. 3. The Nusselt number as a function of time for $\mathrm{Ra}=50$ in a square unit box. Data for $\delta_{M}^{\prime}=0.033$ (solid red line), 0.025 (dashed blue line), and 0.017 (dotted green line).

simulation is stopped when this flux remains constant over a dimensionless period of time at least equal to 1 .

\section{B. Comparison with previous studies}

Other values of $\mathrm{Ra}$ are considered now, in cubic boxes. This work only addresses stable steady-state convection and does not consider transient regimes.

In order to obtain accurate results, the mesh size needs to be chosen as a function of Ra. A sufficiently high level of mesh refinement is necessary in order to avoid oscillations that can be generated in areas with steep temperature gradients. Therefore, the discretization length depends on Ra and on the required accuracy.

It is observed that the Nusselt number decreases when the spatial resolution in the simulations is refined [Fig. 4(a)]. Therefore, using the data for various $\delta_{M}^{\prime}, \mathrm{Nu}_{\text {ref }}$ is calculated as the extrapolation of $\mathrm{Nu}$ for $\delta_{M}^{\prime} \rightarrow 0$. Figure 4(b), where $\Delta \mathrm{Nu}=\left|\mathrm{Nu}-\mathrm{Nu}_{\mathrm{ref}}\right| / \mathrm{Nu}_{\mathrm{ref}}$ is displayed as a function of $\mathrm{Ra}$, illustrates the convergence of $\mathrm{Nu}$ with the discretization. For example, for $\mathrm{Ra} \leqslant 150, \mathrm{Nu}$ is known with a precision better than $2 \%$ if $\delta_{M}^{\prime} \leqslant 0.033$. It is also observed that a finer discretization is required to reach a prescribed precision when Ra increases.

Let us now analyze the convection behavior in order to find the conditions where the fluid starts circulating. It is found that the numerical value of the critical Rayleigh number $\mathrm{Ra}_{c}$ in closed-top conditions ranges between 39.41 and 39.67, values that agree with the theoretical value of $4 \pi^{2} \simeq 39.48$. Then, in order to obtain steady-state convection patterns of various intensities, $\mathrm{Ra}$ is varied from $\mathrm{Ra}_{c}$ to $\mathrm{Ra}=300$.

As discussed at the beginning of Sec. III, steady-state convection can be stable for several types of two-dimensional flow patterns, namely one-, two-, and three-cell convection [39]. In some cases, in order to obtain a particular flow pattern, one has to start from a nonuniform initial state or to increase progressively the temperature. For example, when $\mathrm{Ra}=100$, after an instantaneous and symmetric heating, the system spontaneously evolves to a two-cell convection, with $\mathrm{Nu} \simeq 2.15$. Nevertheless, when unusual initial conditions are imposed, such as perturbations causing a nonsymmetric distribution of the temperature field at $t^{\prime}=0$, a stable steady-state one-cell convection is achieved, with $\mathrm{Nu} \simeq 2.64$ (see Table II). (a)
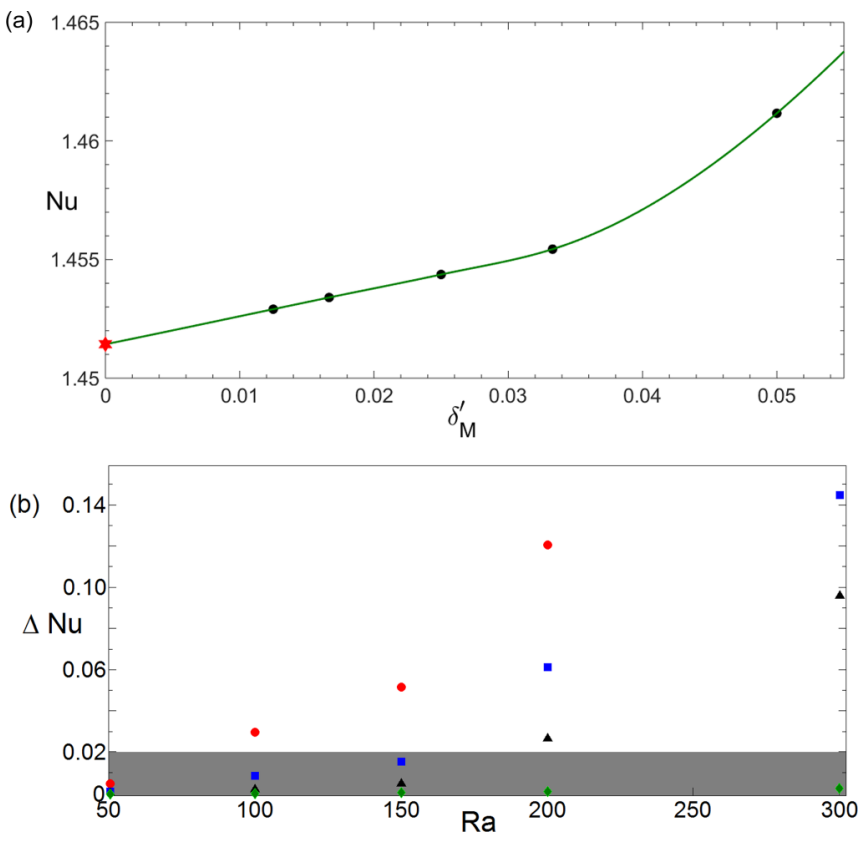

FIG. 4. (a) The Nusselt number as a function of the dimensionless mesh size $\delta_{M}^{\prime} . \mathrm{Ra}=50$. Black dots correspond to simulations; the red star is the extrapolated value $\mathrm{Nu}_{\text {ref }}$, for $\delta_{M}^{\prime}=0$. (b) The deviation $\Delta \mathrm{Nu}=\left|\mathrm{Nu}-\mathrm{Nu}_{\mathrm{ref}}\right| / \mathrm{Nu}_{\mathrm{ref}}$ between our simulations for various $\delta_{M}^{\prime}$, and the extrapolated $\mathrm{Nu}_{\text {ref }}$. Data for $\delta_{M}^{\prime}=0.05$ (red dots), 0.033 (blue squares), 0.025 (black triangles), and 0.017 (green diamonds). The grey area corresponds to errors smaller than $2 \%$.

Our results for $2 \mathrm{D}$ convection patterns obtained in cubic boxes with closed-top conditions and $\mathrm{Ra}$ up to 300 are compared to the numerical data published in Refs. [39,41] in Table II, for one- and two-cell convection. Results for $3 \mathrm{D}$ circulations are shown in Table III and compared with Ref. [39].

Note that only one form of three-dimensional circulation is stable in our realizations, as it was previously highlighted in Ref. [39]. Two upflowing plumes are located along two opposite edges of the cube, and two downflowing plumes are

TABLE II. The Nusselt number for 2D steady-state convection in closed-top conditions for the current study, compared to the data of Refs. [39,41]. The numerical data are the extrapolated Nusselt values for $\delta_{M}^{\prime} \rightarrow 0$. Two-cell convection is indicated by an asterisk; other values are for one-cell convection.

\begin{tabular}{lllr}
\hline \hline & \multicolumn{3}{c}{$\mathrm{Nu}$} \\
\cline { 2 - 4 } Ra & This study & Ref. [39] & Ref. [41] \\
\hline 50 & 1.451 & & 1.450 \\
100 & 2.643 & 2.651 & 2.647 \\
100 & $2.146^{*}$ & & \\
150 & 3.315 & 3.320 & 3.324 \\
150 & $3.251^{*}$ & $3.245^{*}$ & \\
200 & 3.805 & 3.808 & 3.801 \\
200 & $3.992^{*}$ & $3.986^{*}$ & \\
300 & 4.512 & 4.510 & 4.519 \\
300 & $5.024^{*}$ & $5.005^{*}$ & \\
\hline \hline
\end{tabular}


TABLE III. The Nusselt number for 3D steady-state convection in closed-top conditions for the current study (data are extrapolations of $\mathrm{Nu}$ for $\delta_{M}^{\prime} \rightarrow 0$ ) and Ref. [39].

\begin{tabular}{lcc}
\hline \hline & \multicolumn{2}{c}{$\mathrm{Nu}$} \\
\cline { 2 - 3 } $\mathrm{Ra}$ & This study & Ref. [39] \\
\hline 100 & 2.646 & \\
200 & 4.377 & 4.41 \\
300 & 5.38 & 5.43 \\
\hline \hline
\end{tabular}

located along the two other edges. Isotherms present an axial symmetry with respect to the vertical axis passing through the center of the box. The occurrence of this circulation is highly dependent on initial conditions. At least for Ra up to 300 , if the heating is instantaneous and distributed over a horizontal plane, the steady-state convection in a cubic box of homogeneous porous medium is two dimensional (i.e., isotherms are perfectly horizontal along the $x$ or $y$ direction).

In open-top conditions, $\mathrm{Ra}_{c}$ ranges between 26.79 and 27.12 , which is in agreement with the theoretical prediction of 27.10 derived in Refs. [3,4].

Since the numerically obtained Nusselt numbers compare well with those of Refs. [39,41], the current numerical approach is assumed to provide accurate solutions to the closedtop problem for Ra up to 300 .

Simulation results are represented in Fig. 5 for the range $40 \leqslant \mathrm{Ra} \leqslant 150$ together with the polynomial fits

$$
\begin{aligned}
\mathrm{Nu}= & -1.59 \times 10^{-9} \mathrm{Ra}^{4}+1.31 \times 10^{-6} \mathrm{Ra}^{3} \\
& -4.07 \times 10^{-4} \mathrm{Ra}^{2}+6.56 \times 10^{-2} \mathrm{Ra}-0.981, \\
\mathrm{Nu}= & -3.14 \times 10^{-10} \mathrm{Ra}^{4}+3.76 \times 10^{-7} \mathrm{Ra}^{3} \\
& -1.83 \times 10^{-4} \mathrm{Ra}^{2}+5.04 \times 10^{-2} \mathrm{Ra}-0.903,
\end{aligned}
$$

where Eqs. (24a) and (24b) correspond to two- and threedimensional circulation, respectively.

Hence, at higher Rayleigh numbers, 3D Nu is higher than 2D Nu, while the opposite is true at low Ra. This result has been highlighted by others authors, such as in Ref. [39].

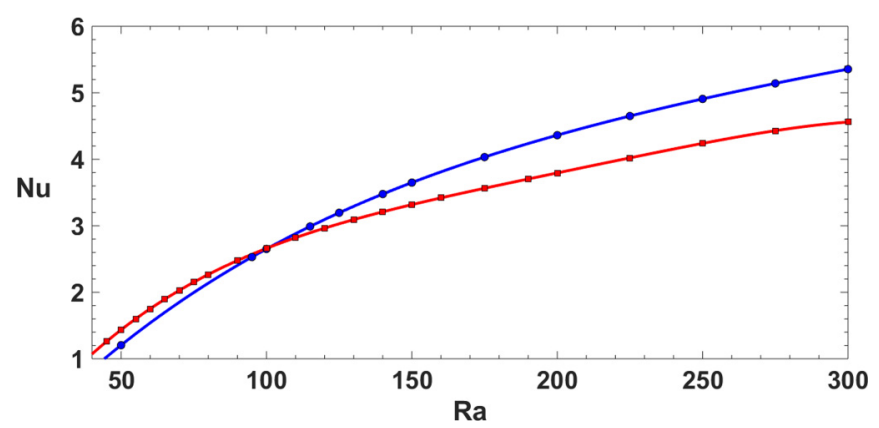

FIG. 5. The Nusselt number as a function of the Rayleigh number Ra. Red squares and blue dots are for simulations of two-dimensional and three-dimensional convection in homogeneous porous media, respectively. Red squares denote two-dimensional convection [Eq. (24a)], and blue circles denote three-dimensional convection [Eq. (24b)].

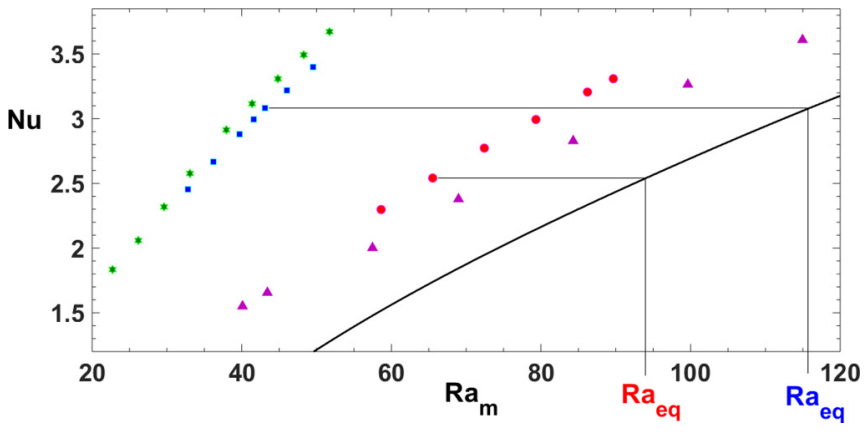

FIG. 6. The Nusselt number as a function of the matrix Rayleigh number $\mathrm{Ra}_{m}$. The solid line is the interpolation (24b). Data for $\rho^{\prime}=$ $2 \sigma^{\prime}=1$ (purple triangles), $\rho^{\prime}=3$ and $\sigma^{\prime}=5$ (green stars), $\rho^{\prime}=4$ and $\sigma^{\prime}=1$ (red dots), and $\rho^{\prime}=7$ and $\sigma^{\prime}=2$ (blue squares).

It was proposed in Ref. [42] that three-dimensional motion consists of a superposition of orthogonal rolls, suggesting that two-dimensional circulation transfers more heat for Rayleigh numbers close to $\mathrm{Ra}_{c}$, but there has been no rigorous demonstration of this suggestion.

\section{THERMAL CONVECTION IN FRACTURED POROUS MEDIA}

In this section, the influence of a fracture network on the magnitude of thermal convection is studied in cubic boxes with $R^{\prime}=0.2$. The magnitude of the convection obviously depends on the Rayleigh number in the matrix $\operatorname{Ra}_{m}$ [the subscript $m$ is a reminder that $\mathrm{Ra}_{m}$ is evaluated with the matrix permeability $K_{m}$ in Eq. (14)] and on the characteristics of the fracture network.

According to simulations in homogeneous porous media (see Sec. IIIB), the precision of the results improves with decreasing $\delta_{M}^{\prime}$ [cf. Eq. (23d)]. It has been numerically observed that the same is verified for fractured media. The value $\delta_{M}^{\prime}=$ $1 / 30$ was chosen as a trade-off between computation time and $\mathrm{Ra}$ range; therefore, all computations where $\mathrm{Ra}>160$ ( $\mathrm{Nu} \gtrsim 4$ for three-dimensional flow patterns) are excluded. For the same practical reasons, the maximum values of $\rho^{\prime}$ and $\sigma^{\prime}$ are $10 ; \rho^{\prime}>10$ implies large computation times, while $\sigma^{\prime}>10$ necessitates grid resolution better than the value of $\delta_{M}^{\prime}$ used in this study in order to provide accurate results.

As discussed in Sec. II C, $\Lambda^{\prime} \ll 1$ and $\omega^{\prime} \ll 1$ in all cases. In other words, owing to the moderate contrast of the fluid and solid thermal properties and to their very small volume fraction, the fractures have a negligible impact on the thermal conduction and inertia. Hence, the fracture contribution is mostly convective and their influence on the heat released by the system primarily depends on $\sigma^{\prime}$ and $\rho^{\prime}$.

In Fig. 6, $\mathrm{Nu}$ is displayed as a function of $\mathrm{Ra}_{m}$ for four different fracture networks with various $\rho^{\prime}$ and $\sigma^{\prime}$. The Nusselt number for stable steady-state three-dimensional convection in cubic homogeneous porous media [Eq. (24b)] is also plotted. The heat transfer in fractured porous media is enhanced relative to that in homogeneous porous media for the same boundary conditions due to the presence of fractures. The output flux characterized by $\mathrm{Nu}$ strongly depends on the structural and hydraulic properties $\rho^{\prime}$ and $\sigma^{\prime}$. 

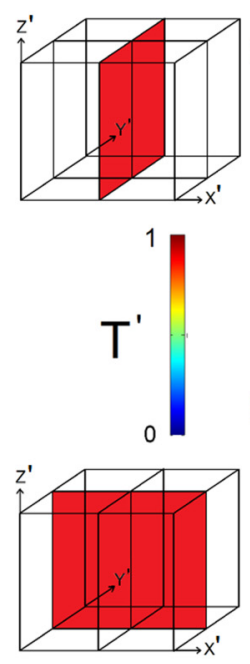

(a)

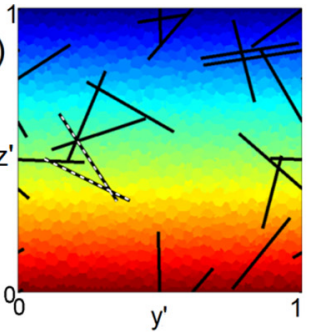

(d)

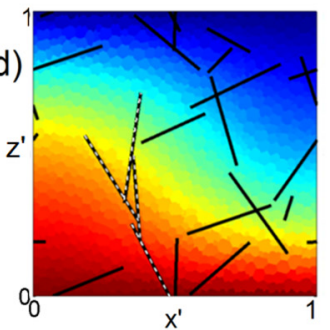

(b)

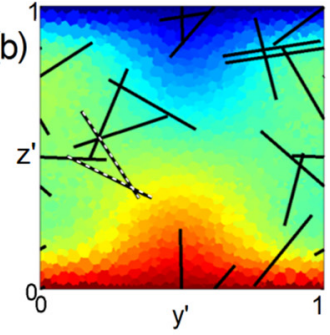

(e)

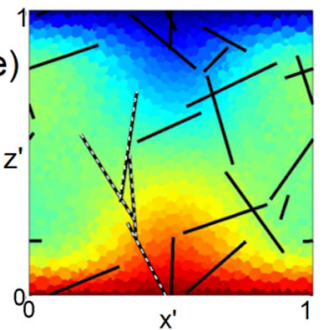

(c)
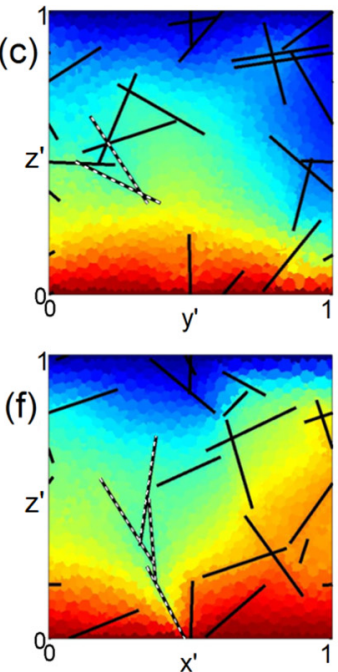

FIG. 7. The temperature field in the two orthogonal vertical midplanes at steady state for three different dimensionless fracture transmissivities: (a), (d) $\sigma^{\prime}=0.1$; (b), (e) 1; and (c), (f) 5. Data for $\rho^{\prime}=4$ and $\mathrm{Ra}_{m}=41.5$.

Figure 6 shows that the data for each network vary smoothly with $\mathrm{Ra}_{m}$, with a trend similar to that for the homogeneous porous medium. An equivalent Rayleigh number $\mathrm{Ra}_{\mathrm{eq}}$ is defined as the Rayleigh number for which the unfractured porous medium releases the same thermal flux.

The rest of this section is divided into three parts. The two first ones address the influence of the dimensionless transmissivity and of the dimensionless fracture density on the heat released by an isotropically fractured system. The third part discusses the calculation of the effective permeability of a fractured medium and compares the output fluxes generated by the discrete fractured approach and the associated effective medium approach.

\section{A. Influence of the dimensionless fracture transmissivity}

The influence of $\sigma^{\prime}$ on the natural thermal convection in a cubic box is illustrated by vertical cross sections of steady-state temperature fields (Fig. 7) in a given fractured porous medium with $\rho^{\prime}=4$. The matrix Rayleigh number $\mathrm{Ra}_{m}$ is kept constant and equal to 41.5 .

In order to schematize the results, the flow will often be said to be two dimensional when the mean $x$ component of the velocity is about one order of magnitude lower than the $y$ and $z$ components. This is clearly approximate since when random fractures are present, the flow is necessarily three dimensional.

In accordance with Rayleigh's theory, if Ra is larger than $\mathrm{Ra}_{c}$, convection occurs with the formation of at least one convection cell [39]. As noticed in Sec. III, for an instantaneous and horizontally distributed heating from below, the steady-state convection in a homogeneous porous medium is two dimensional, at least for $\mathrm{Ra}<300$. If the medium is fractured, this is no longer true since the medium is not homogeneous anymore. However, for small fracture conductivities [e.g., $\sigma^{\prime}=0.1$ in Figs. 7(a) and 7(d)], the convection patterns have a pronounced two-dimensional structure, with perfectly horizontal isotherms along the $x$ direction. In that case, fractures do not significantly perturb the medium and the steady-state temperature field indeed presents a pronounced two-dimensional structure.

The temperature fields shown for $\sigma^{\prime}=1$ and 5 in Figs. 7(b)7 (f) correspond to three-dimensional convective regimes. For $\sigma^{\prime}=1$ [Figs. 7(b) and 7(e)], the temperature field at steady state presents a symmetry around the $z$ axis passing through the center of the box, which reminds one of three-dimensional convection in a cubic box of homogeneous porous medium (see Sec. III B). For larger $\sigma^{\prime}$ [Figs. 7(c) and 7(f)], the steady-state temperature field is highly influenced by the fracture location.

Obviously, the flow patterns in a given fracture network vary with the fracture transmissivity. For example, consider the fractures with white dashes in Figs. 7(a)-7(f). As one can notice by comparing the temperature field surrounding these fractures, some upflowing fractures turn into downflowing fractures when their transmissivity is increased.

The variations of the output flux are illustrated in Fig. 8, where $\mathrm{Nu}$ is plotted as a function of $\sigma^{\prime}$, for $\mathrm{Ra}_{m}=41.5$ and four individual networks with densities $\rho^{\prime}=1,4$, and 8 .

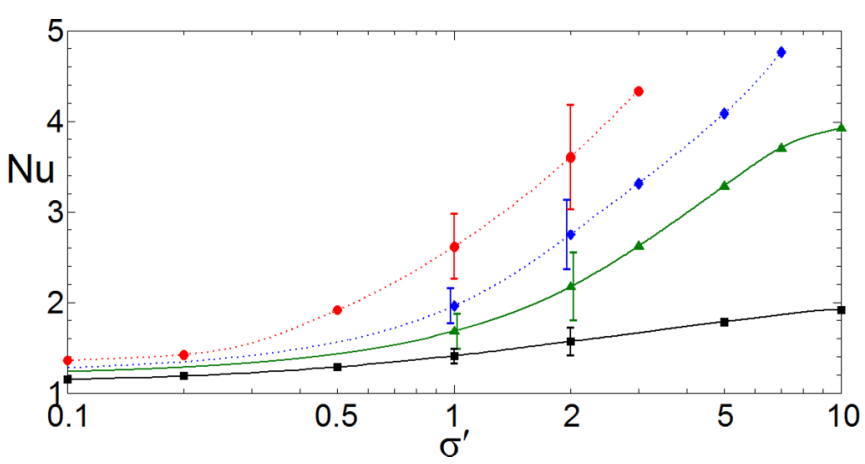

FIG. 8. The Nusselt number as a function of the dimensionless fracture transmissivity $\sigma^{\prime}$. Data for $\rho^{\prime}=8$ (red circles), 4 (blue diamonds and green triangles), and 1 (black squares); $\mathrm{Ra}_{m}=41.5$. Solid and dashed lines correspond to small and large $\mathrm{Nu}$ increments, respectively. The error bars correspond to $\pm 2 \sigma_{d}, \sigma_{d}$ being the standard deviation observed over a set of 18 realizations (Fig. 10). 

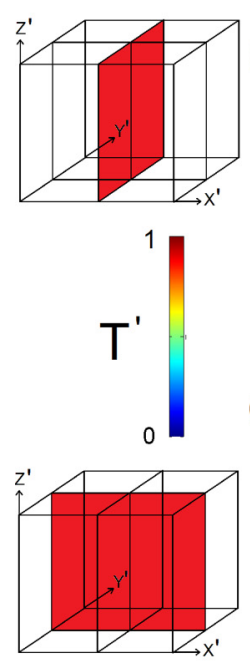

(a)

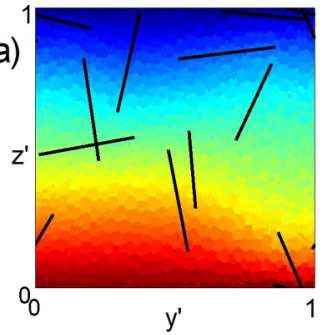

(d)

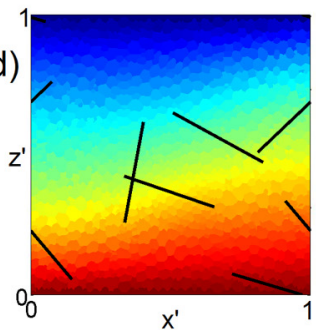

(b)

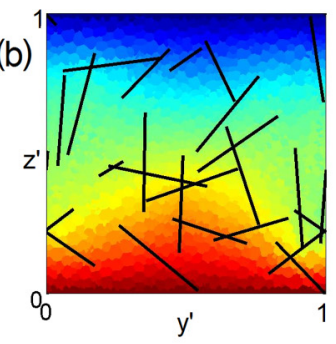

(e)

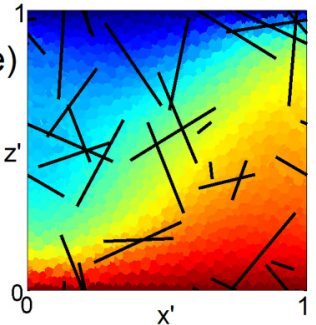

(c)
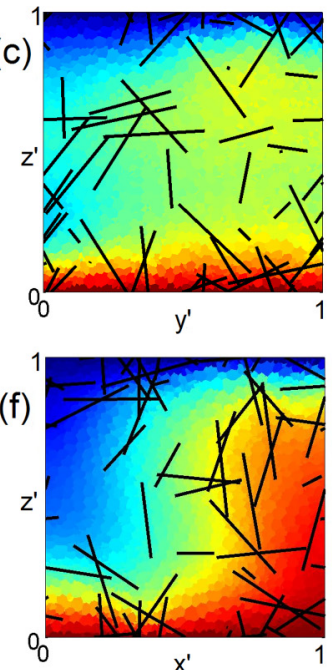

FIG. 9. The temperature field in two perpendicular vertical midplanes at steady state for (a), (d) $\rho^{\prime}=2$, (b), (e) $\rho^{\prime}=6$, and (c), (f) $\rho^{\prime}=10$. Data for $\sigma^{\prime}=1$ and $\mathrm{Ra}_{m}=41.5$.

The trend of $\mathrm{Nu}$ as a function of $\sigma^{\prime}$ depends on the fracture networks. Data for $\mathrm{Nu}>4$ are obtained with a relatively low precision (error $\geqslant 2 \%$ ). As seen in Fig. 8, two different behaviors are depicted for $\sigma^{\prime}$ varying from 0.1 to 10 . In networks with small densities (e.g., $\rho^{\prime}=1$ ), the increase of $\mathrm{Nu}$ is very limited for large $\sigma^{\prime}$, which is not true for largedensity networks (e.g., $\rho^{\prime}=8$ ). For moderate densities such as $\rho^{\prime}=4$, both trends can be observed. A similar behavior was observed for the permeability of a fracture network as a function of $\sigma^{\prime}$ [29]. More precisely, when the fracture network does not percolate, the fluid has to flow through the porous matrix. Therefore, an increase in $\sigma^{\prime}$ has a limited effect on the effective permeability. In the opposite case, when the network percolates, the effective permeability of the medium increases proportionally to $\sigma^{\prime}$. However, because of the low precision, the existence of a plateau for low $\rho^{\prime}$ and large $\sigma^{\prime}$ cannot be demonstrated.

One can suppose that a similar mechanism determines the convective behavior of fractured media. If the permeability of the matrix is fixed, and the fracture network does not percolate, increasing the permeability of the fractures enhances convection in a limited way, because the fluid has to flow through the matrix. However, the concept of percolation for thermal convection in closed boxes should be different from that for a linear flow, because the fluid follows the pattern of the convection cell, not only circulating from one face of the box to the opposite one.

Nevertheless, these behaviors could not be checked for $\sigma^{\prime}>$ 10 because a better discretization is necessary.

\section{B. Influence of the dimensionless fracture density $\rho^{\prime}$}

The influence of $\rho^{\prime}$ on the natural thermal convection in a cubic box is illustrated in Fig. 9 by steady-state temperature fields obtained for various $\rho^{\prime}$ and fixed values of $\mathrm{Ra}_{m}=41.5$ and $\sigma^{\prime}=1$.

Structural transitions of the convective circulation are observed. For a low-density fracture network [Figs. 9(a) and 9(d)], which does not percolate, the observed convection does not differ significantly from that in homogeneous porous media. The slope of the isotherms along the $x$ and $y$ directions is small. When $\rho^{\prime}$ is increased, the convection structure changes [Figs. 9(b) and 9(e)]. The convection patterns present inclined isotherms along the $y$ direction, and a central upflowing plume is visible along the $x z$ plane. In the dense fracture network displayed in Figs. 9(c) and 9(f), the flow pattern is almost two dimensional, with a well-defined upflowing plume along the $y z$ plane, and with quite flat isotherms along the $x z$ plane. A strong difference in convection strength differentiates lowand high-density networks, since the lateral extension of the upflowing plume is thinner when $\rho^{\prime}$ is increased.

In Fig. 10, Nu is displayed as a function of $\rho^{\prime}$, for a fixed $\mathrm{Ra}_{m}$ and two different fracture transmissivities, namely, $\sigma^{\prime}=1$ and 2. The data are statistical means over 18 different networks. The standard deviations represented by the error bars increase with increasing $\rho^{\prime}$; they are approximately equal to $20 \%$ of the mean $\mathrm{Nu}$.

The two transmissivities, $\sigma^{\prime}=1$ and 2 , are not sufficiently large to observe different behaviors according to the percolating character of the networks tested (see Fig. 8). The trend of

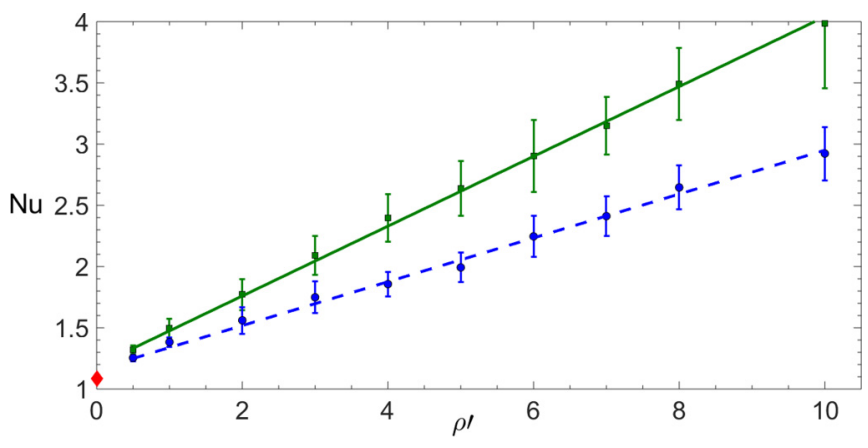

FIG. 10. The average Nusselt number as a function of the dimensionless fracture density $\rho^{\prime}$ for $\sigma^{\prime}=1$ (blue circles), $\sigma^{\prime}=2$ (green squares), and $\mathrm{Ra}_{m}=41.5$ (the red star represents $\mathrm{Nu}_{\text {mat }}$ ). Solid and broken lines are the linear fits [Eqs. (25a) and (25b)]. 
$\mathrm{Nu}$ as a function of $\rho^{\prime}$ is quite linear for the ranges of $\rho^{\prime}$ and $\sigma^{\prime}$ tested. Tentative approximation fits yield

$$
\begin{array}{ll}
\mathrm{Nu}=1.19+0.285 \rho^{\prime} & \text { for } \quad \sigma^{\prime}=2, r=0.999, \\
\mathrm{Nu}=1.16+0.179 \rho^{\prime} & \text { for } \quad \sigma^{\prime}=1, r=0.996 .
\end{array}
$$

In the no-fracture limit, $\rho^{\prime}=0, \mathrm{Nu}$ is equal to $\mathrm{Nu}_{\text {mat }}=$ 1.087, represented in Fig. 10. This value does not verify the fits in Eqs. (25), but still remains in the $\pm 20 \%$ error bar.

\section{Convection in fractured porous media and in effective homogeneous porous media}

In this section, the heat released by the fractured porous medium is compared to the heat released by a homogeneous porous medium with the same effective permeability. The results obtained with the fractured porous medium are denoted by the subscript FPM while results obtained with the corresponding homogeneous porous medium are denoted by the subscript HPM.

\section{Computation of the effective properties of fractured porous media}

The influence of fractures on the effective properties of the whole medium depends on their density and their thermal and hydraulic transmissivities. In this study, the presence of fractures increases the macroscopic permeability, as fractures present a higher permeability than the porous matrix, but does not change the thermal conductivity of the medium, as noticed at the beginning of Sec. IV A. Therefore, the effective thermal conductivity of the fractured medium is identical to the thermal conductivity $\lambda_{m}$ of the porous matrix. The fracture presence does not significantly modify the global heat capacity either, which is considered equal to the heat capacity of the porous matrix.

To obtain the effective macroscopic permeability, a procedure analogous to that in Ref. [30] is followed. First, for each sample of fractured porous medium, a pressure drop is imposed between two opposite boundaries while the other boundaries are kept impervious. Then, the average fluid velocity is calculated without considering the heat transfer. The relation between this velocity and the imposed pressure gradient is used in order to evaluate the sample permeability via the macroscopic Darcy law. Simulations are done along the three spatial directions and the effective permeability of the isotropic fractured porous medium $K_{\text {eff }}$ is the mean of the three diagonal values. Once $K_{\text {eff }}$ is computed for a given network, fractures are removed, and the permeability of the matrix is replaced by $K_{\text {eff }}$. Then, simulations of convection in this effective medium are performed for the same boundary conditions as for the fractured medium.

\section{Output thermal flux}

The following results are obtained for fractured media when $\rho^{\prime}$ varies from 0.5 to 10 and $\sigma^{\prime}$ from 0.1 to 10 . The Rayleigh number $\mathrm{Ra}_{m}$ is changed by varying $\Delta T$. The interval of variations of $\Delta T$ is limited to values for which the effective Rayleigh number $\mathrm{Ra}_{\text {eff }}$, evaluated by Eq. (14) with $K_{m}$ replaced by $K_{\text {eff }}$, does not exceed 150 .

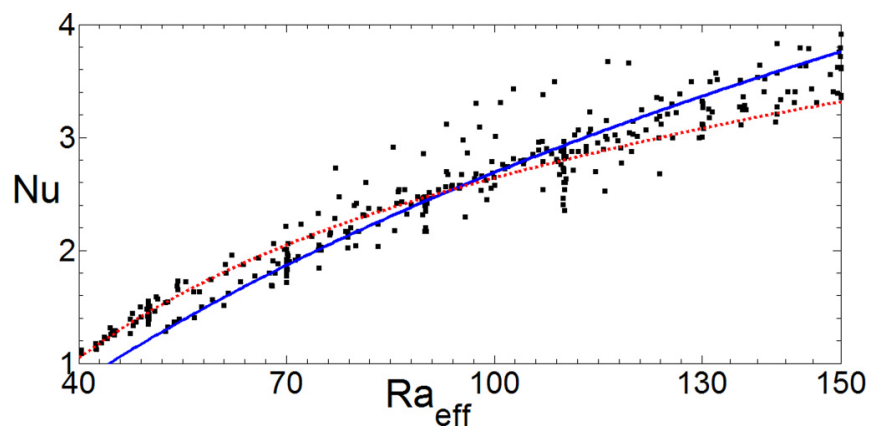

FIG. 11. The Nusselt number as a function of $\mathrm{Ra}_{\text {eff }}$. Black dots represent $\mathrm{Nu}$ with the FPM approach for $0.1 \leqslant \sigma^{\prime} \leqslant 10,0.5 \leqslant \rho^{\prime} \leqslant$ 10 , and $7 \leqslant \mathrm{Ra}_{m} \leqslant 139$. The dotted red line and solid blue line stand for two- and three-dimensional convection in a HPM.

In order to evaluate the efficiency of the homogeneous approach without any constraint on the topology of the fracture network, Fig. 11 shows the Nusselt number obtained in a FPM as a function of $\mathrm{Ra}_{\text {eff }}$ compared to the values of $\mathrm{Nu}$ for two- and three-dimensional convection in a HPM as given by Eqs. (24). This graph should be compared with Fig. 6, where $\mathrm{Nu}$ is given as a function of $\mathrm{Ra}_{m}$ for some networks. In a first approximation, the data are well gathered when $\mathrm{Ra}_{\mathrm{eff}}$ is used instead of $\mathrm{Ra}_{m}$ and they are close to $\mathrm{Nu}$ for homogeneous porous media. However, some scatter can be observed as well as particular trends, which are going to be analyzed. Note that according to Fig. 4 the numerical errors are kept smaller than $2 \%$.

In order to obtain a precise relationship between an average Nusselt number and $\mathrm{Ra}_{\mathrm{eff}}$, a systematic statistical study should be performed. A large number of networks should be generated for a given density and a given aperture. Then, $\mathrm{Nu}$ should be determined for each fractured porous medium and each $\mathrm{Ra}_{\mathrm{eff}}$. Considering the duration of the computations, this was not undertaken here, but it can be said that the statistical scattering is similar to the one observed in Fig. 8.

As discussed in Sec. IV A and demonstrated in Fig. 7, a stable convective flow in fractured porous media can be almost two dimensional or three dimensional under the same thermal conditions, depending on the characteristics of the fracture network. In a homogeneous porous medium, the convection pattern can also be two or three dimensional, depending on the initial conditions or on the imposed boundary conditions. These patterns correspond to different Nusselt numbers; hence, the FPM results have to be compared to the HPM results with a similar flow pattern. In order to select the FPM simulations which should be compared with HPM two-dimensional convection and to separate them from simulations which should be compared to HPM three-dimensional convection, the volume averages of the absolute value of the fluid velocities along the $x$ and $y$ directions are calculated. When these velocities differ by one order of magnitude or more, the FPM convection is said to be two dimensional.

Figure 12 represents $\mathrm{Nu}_{\mathrm{HPM}}$ as a function of $\mathrm{Nu}_{\mathrm{FPM}}$, with the same effective permeability. The comparison takes into account the convection structure, which is either two or three dimensional. It is useful to separately analyze two- and three- 


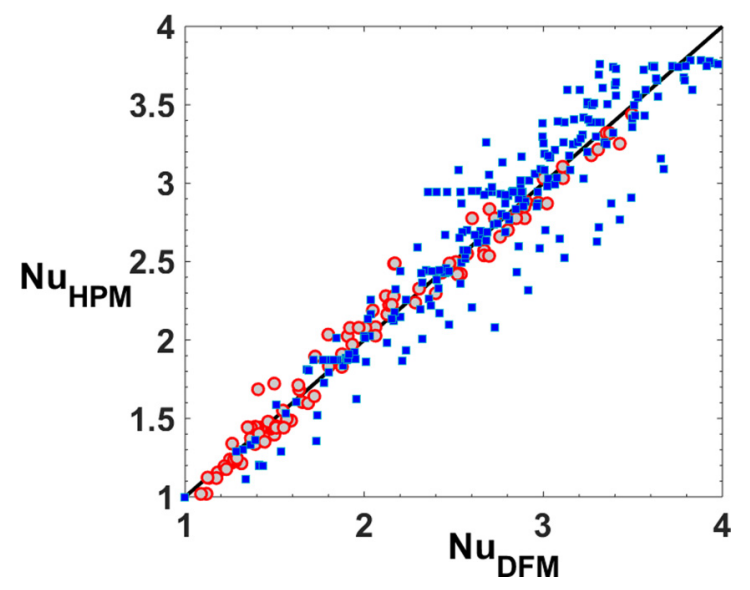

FIG. 12. The Nusselt number $\mathrm{Nu}_{\mathrm{HPM}}$ computed in a homogeneous porous medium vs the Nusselt number $\mathrm{Nu}_{\mathrm{FPM}}$ computed in the discrete fracture medium, both presenting the same effective permeability. Data for two-dimensional convection (red dots) and three-dimensional convection (blue squares); one dot represents one realization, and the first bisector is shown. Additional data: $7<$ $\mathrm{Ra}_{m}<139,0.5<\rho^{\prime}<10$, and $0.1<\sigma^{\prime}<10$.

dimensional FPM flow patterns because they demonstrate different tendencies.

Two-dimensional convection patterns present three different trends. The first regime concerns weak $\mathrm{Nu}<1.4$, when $\mathrm{Ra}_{\text {eff }}$ is close to $\mathrm{Ra}_{c}$. In this case, the HPM tends to underestimate the output flux of the FPM; therefore, fractures, when present in the numerical model, are more influential than expected. For $1.4 \leqslant \mathrm{Nu} \leqslant 2.7$, the two-dimensional convection in HPM tends to overestimate the output flux and to underestimate it for $\mathrm{Nu} \geqslant 2.7$. This change is probably due to the fact that two-dimensional convection in cubic boxes transfers more heat than three-dimensional convection for $\mathrm{Ra} \leqslant 100$ [Eqs. (24)], corresponding to $\mathrm{Nu} \simeq 2$.7. Since the medium is not homogeneous, convection cannot be perfectly two dimensional. In our simulations, the horizontal velocities never vanish, and the two-dimensional patterns are characterized by a large velocity difference between the $x$ and $y$ directions. Hence, the convection patterns in FPM have a hybrid structure between two- and three-dimensional convections, leading to an overestimation by HPM for $\mathrm{Nu} \leqslant 2.7$ and underestimation for $\mathrm{Nu} \geqslant 2.7$. It is observed that the ratio between velocities along the $y$ and $x$ directions does not vary significantly when $\mathrm{Ra}_{m}$ increases, since velocities along all directions increase.

The comparison between HPM and FPM for threedimensional flow patterns is less intuitive as no obvious trend appears. For $\mathrm{Nu} \leqslant 1.5$, HPM systematically underestimates the output flux generated by FPM; again the influence of fractures is larger than expected when $\mathrm{Ra}_{\text {eff }}$ is weak. When $\mathrm{Nu}$ is increased, no obvious trend is seen; HPM can overestimate or underestimate the heat released by FPM. Among 239 simulations in FPM compared to three-dimensional convection in HPM, 124 simulations exhibit an overestimation of HPM. As can be seen in Figs. 11 and 12, some data points are perfectly aligned, with $\mathrm{Nu}_{\mathrm{HPM}}=1.44,1.87,2.94$, and 3.75 , and correspond to simulations with $\mathrm{Ra}_{\mathrm{eff}}=50,70,110$, and 150 , respectively. These simulations are relative to networks with various $\rho^{\prime}$ and various $\sigma^{\prime}$. The fact that these dots are not superimposed and that $\mathrm{Nu}_{\mathrm{FPM}}$ varies from one network to another means that the applicability of the HPM depends on the network parameters.

\section{DISCUSSION}

\section{A. Validity of the homogeneous approach}

As demonstrated in Sec. IV, some predictions of HPM based on $K_{\text {eff }}$ provide the same macroscopic heat transfer as in the corresponding FPM; nevertheless, for several networks, HPM provides contrasts with FPM larger than $25 \%$. Let us analyze the error of HPM as a function of the parameters tested in this study, namely, the network density $\rho^{\prime}$ and the transmissivity $\sigma^{\prime}$ of the fractures.

Figure 13 represents the same data as in Fig. 12, in four panels according to the value of $\rho^{\prime}$. Figure 13(a) shows that the effective approach is in agreement with the discrete fracture approach for very low fracture densities, i.e., $\rho^{\prime} \leqslant 2$. Note that these networks are not percolating. The deviation of $\mathrm{Nu}_{\mathrm{HPM}}$ from $\mathrm{Nu}_{\mathrm{FPM}}$ for low-density fracture networks is seen to increase with increasing Ra. Figures 13(b) and 13(c) display results for $3 \leqslant \rho^{\prime} \leqslant 7$; these densities obviously present the largest deviations, up to $29 \%$ between HPM and FPM. Then, Fig. 13(d) corresponds to large-density networks with $\rho^{\prime} \geqslant$ 8 and exhibits lower deviations. In this latter case, HPM predictions become more precise as the heterogeneity of the fractured porous medium decreases.

The fact that HPM is more precise for low and for dense fracture networks is in accordance with physical intuition. For isotropic networks in a cubic domain with $R^{\prime}=0.2$, the probability of percolation reaches $1 / 2$ when $\rho^{\prime} \simeq 2.9$ [43]. In fracture networks with low densities, where few fractures actually intersect without forming a percolating network, the influence of fractures is only due to local disturbances in the flow field around each fracture and $\mathrm{Nu}$ can be approximated by its value in a homogeneous medium, within $6 \%$ (for $\mathrm{Ra}_{\text {eff }}$ up to 110). On the other hand, dense fracture networks are similar to homogeneous media. Perturbations of the flow field are still localized in small domains but are distributed over the whole medium. This last result was already emphasized in Ref. [44] for two-dimensional fractured media. For low and dense fracture networks, the heat released by the fractured porous medium is close to that released by a homogeneous medium presenting the same macroscopic properties within $10 \%$, at least for $\mathrm{Ra}_{\text {eff }}$ up to 150 .

Furthermore, $\mathrm{Ra}_{\text {eff }}$ can be evaluated using predictions of the macroscopic permeability of fractured porous media. The macroscopic permeability $K_{\text {eff }}^{\prime}=K_{\text {eff }} / K_{m}$ of porous media containing dilute and dense networks is given by $[29,45]$

$$
\begin{gathered}
K_{\mathrm{eff}}^{\prime}=1+\kappa_{d} \rho^{\prime}+\kappa_{d}{ }^{2} \rho^{\prime 2} \quad \text { for } \rho^{\prime} \leqslant 2, \\
K_{\mathrm{eff}}^{\prime}=1+\frac{2}{9} \rho^{\prime} \sigma^{\prime}\left[1-\frac{1}{1+\frac{7}{3} \sigma^{\prime-0.7}}\left(1-\frac{\beta_{K} \Delta \rho^{\prime 2}}{\rho^{\prime}\left(1+\beta_{K} \Delta \rho^{\prime}\right)}\right)\right] \\
\text { for } \rho^{\prime} \geqslant 4,
\end{gathered}
$$



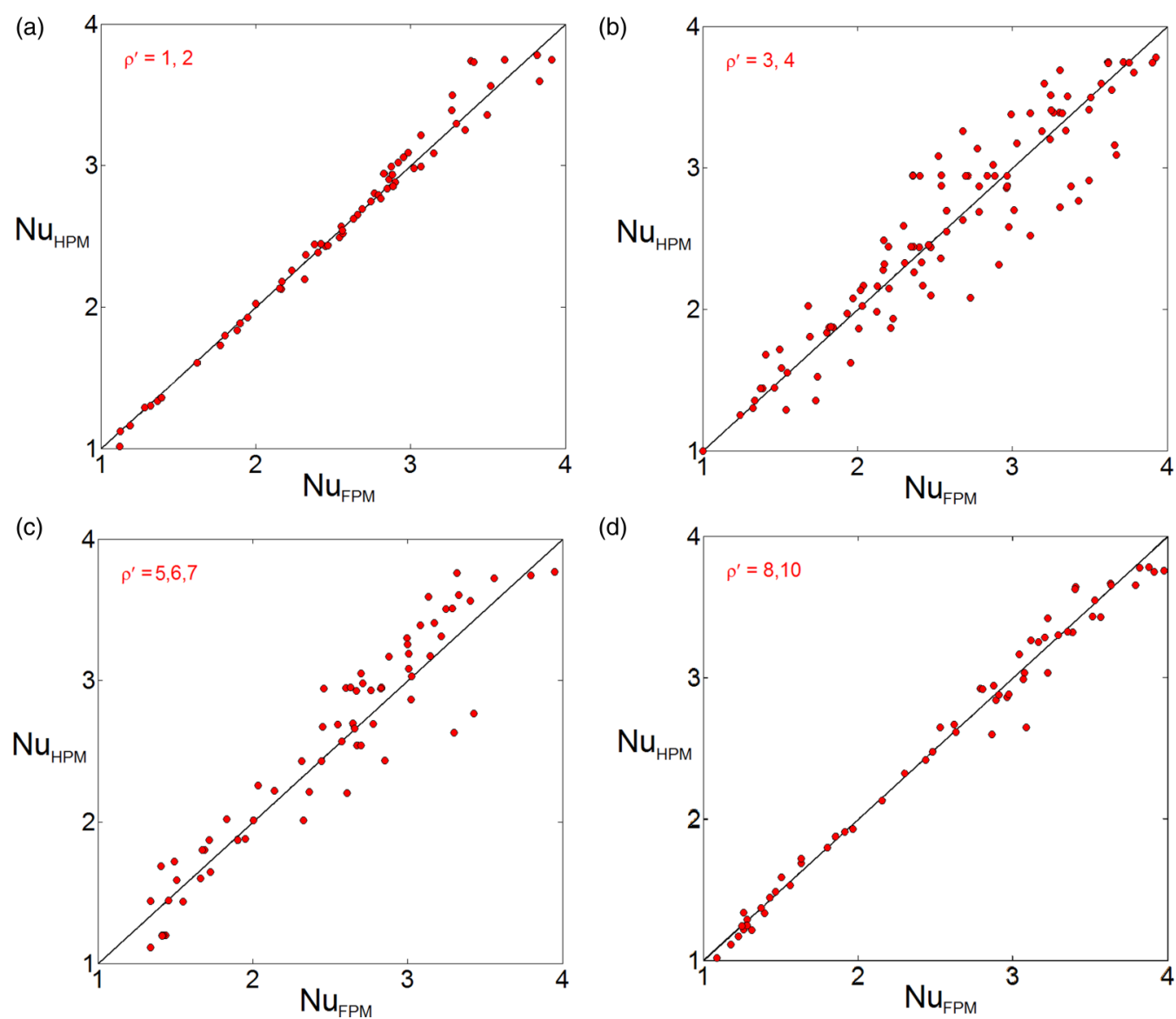

FIG. 13. The Nusselt number $\mathrm{Nu}_{\mathrm{HPM}}$ computed in a homogeneous porous medium as a function of the Nusselt number $\mathrm{Nu}_{\mathrm{FPM}}$ computed in the discrete fracture medium with the same effective permeability. One dot represents one realization, and the first bisector is added for clarity. Data for (a) $\rho^{\prime}=1$ and 2, (b) $\rho^{\prime}=3$ and 4, (c) $\rho^{\prime}=5,6$, and 7, and (d) $\rho^{\prime}=8$ and 10 .

where $\kappa_{d}$ is deduced from

$$
\kappa_{d}=\frac{\sigma^{\prime}}{\sigma^{\prime}+3 / 2} \kappa_{d \infty}
$$

with $\kappa_{d \infty}=0.335, \beta_{K}=0.155$, fitting parameters, and $\Delta \rho^{\prime}=$ $\rho^{\prime}-\rho^{\prime}{ }_{c}$ with $\rho^{\prime}{ }_{c}$ the percolation threshold.

These expressions can be used to determinate $\mathrm{Ra}_{\mathrm{eff}}$, and then $\mathrm{Nu}$ can be evaluated from Eqs. (24). Figure 14 shows the predicted $\mathrm{Nu}$ [Eqs. (24), and $R a_{\text {eff }}$ from Eq. (26)] in comparison with the results obtained in Sec. IV B.

As seen in Fig. 14, the predictions based on Eqs. (24) and (26) agree with the numerical data for FPM within their statistical error bars when $\rho^{\prime} \leqslant 2$ and $\rho^{\prime} \geqslant 4$, for $\operatorname{Ra}_{m}=41.5$ and $\sigma^{\prime}=1$. In addition, since Eqs. (24) apply for Ra up to 300 and Eqs. (26) for any $\sigma^{\prime}$, their combination can be expected to provide reasonable predictions of $\mathrm{Nu}$ at least for the investigated $\mathrm{Ra}$ range. This includes at least the cases in Figs. 13(a) and 13(d).

Let us now consider the effect of the dimensionless transmissivity or aperture of fractures. It was already emphasized in Ref. [44] that fractured systems with uniform aperture systems behave more like homogeneous media than nonuniform aperture systems. Nevertheless, even when fractures present the same transmissivity, the value of this transmissivity is a parameter influencing the validity of the homogenization. This is illustrated in Fig. 15, which presents the deviation $\Delta \mathrm{Nu}$ between the HPM and FPM approaches for one network with $\rho^{\prime}=4$.

$\Delta \mathrm{Nu}$ is seen to increase with $\sigma^{\prime}$. Increasing $\sigma^{\prime}$ implies a relative reduction of the contribution of the matrix to the flow. Therefore, under such conditions, the temperature field deviates from the stable three-dimensional convection structure in

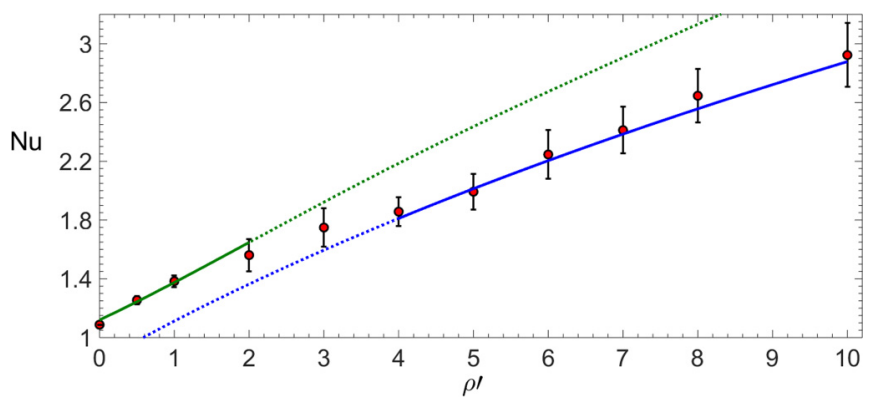

FIG. 14. The Nusselt number as a function of the dimensionless density of fractures, $\rho^{\prime}$, for $\sigma^{\prime}=1$ (red dots) and $\mathrm{Ra}_{m}=41.5$. Dots represent the statistical average over 18 different networks with the same $\rho^{\prime}$. Error bars represent the standard deviations. Solid green and dotted blue curves are the predictions for $\mathrm{Nu}$ deduced from Eqs. (24a) and (26a) and Eqs. (24b) and (26b), respectively, with solid and dotted lines in their range of validity. 


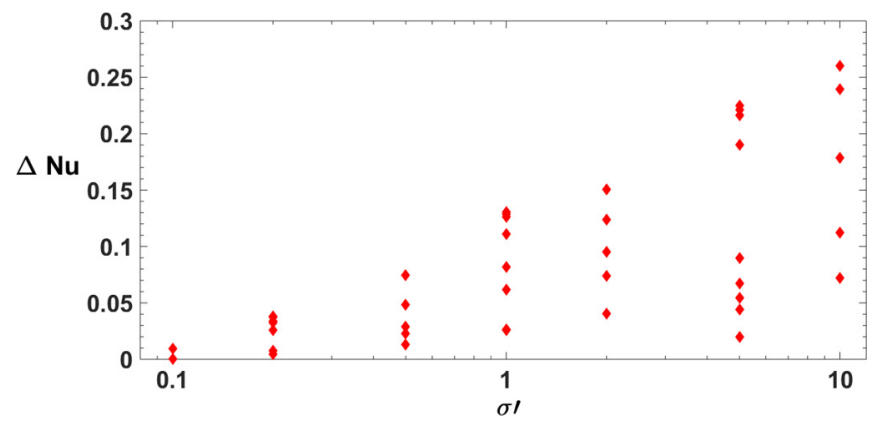

FIG. 15. The deviation $\Delta \mathrm{Nu}=\left|N u_{\text {FPM }}-N u_{\text {HPM }}\right| / N u_{\text {FPM }}$ between fractured and homogeneous porous media with the same effective permeability as a function of the dimensionless transmissivity of fractures $\sigma^{\prime}$ for a density $\rho^{\prime}=4$ and $12<\mathrm{Ra}_{m}<138$. Each data point corresponds to one realization.

cubic homogeneous porous materials, and this effect depends on fracture locations.

These geometrical effects explain the occurrence of the largest deviations between homogeneous and heterogeneous approaches for moderate fracture densities. Nevertheless, it should be noted that the large range of $\rho^{\prime}\left(2 \leqslant \rho^{\prime} \leqslant 7\right)$, inducing potentially inaccurate predictions from HPM, depends on $R^{\prime}$. Therefore, the well-known finite size effect may play a role [46]. As shown in Ref. [30], the smaller the $R^{\prime}$, the sharper the percolation transition. However, with the value $R^{\prime}=0.2$ used in this study, the transition takes place over a wide density interval. The probability $P_{p}$ of percolation for the flow problem increases from 0.05 to 0.95 in the range $1.8 \lesssim \rho^{\prime} \lesssim 4$ [43].

To summarize, the effective approach gives good results when it provides a good estimation of the heat released by the fracture system. This happens when the fracture network does not impact the flow patterns. Since only isotropic networks are studied, two cases meet this requirement. The first one is a nonpercolating fracture network, where only few fractures disturb the convective patterns. The second one is a largedensity fracture network, since the influence of fractures on convective flow patterns decreases with decreasing fracture spacing [37]. Using the effective permeability deduced from Eqs. (26), the Nu can be deduced from Eqs. (24) within $20 \%$ except in the range $2<\rho^{\prime}<4$. Whatever $\rho^{\prime}$, HPM gives better results with decreasing transmissivity. The impact of some geometrical effects are described in Ref. [37] for simplified regular fracture networks.

\section{B. On the existence of an equivalent Rayleigh number}

As shown in Sec. V A, the heat released by a fractured porous medium could be deduced within $20 \%$ by means of HPM. This section aims at understanding if a better precision can be obtained with HPM.

Figure 6 displays $\mathrm{Nu}$ as a function of $\mathrm{Ra}_{m}$ for various networks. The evolution of $\mathrm{Nu}$ with increasing $\mathrm{Ra}_{m}$ is smooth and data are aligned along curves analogous to the ones of homogeneous porous media. Note that the trends of networks $\rho^{\prime}=3, \sigma^{\prime}=5$ and $\rho^{\prime}=7, \sigma^{\prime}=2$ are very similar, even if these networks are morphologically different, with different effective permeabilities ( $K_{\text {eff }}^{\prime}=2.25$ and 3.02, respectively).

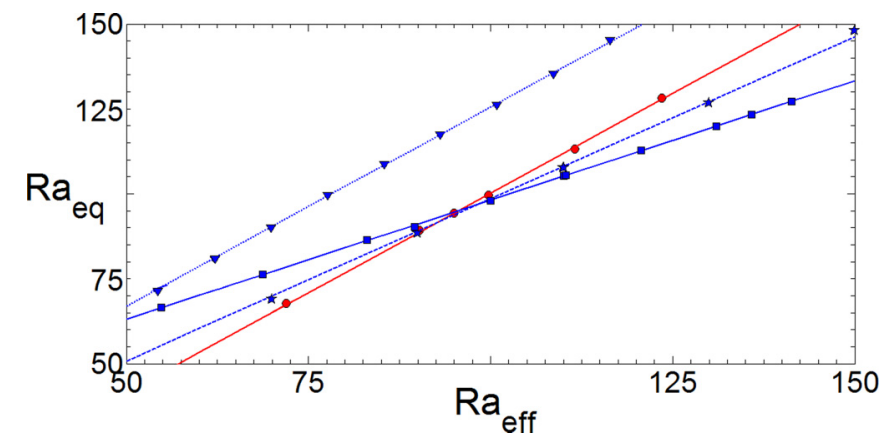

FIG. 16. The equivalent Rayleigh number $\mathrm{Ra}_{\mathrm{eq}}$ as a function of the effective Rayleigh number $\mathrm{Ra}_{\text {eff }}$ for four individual fracture networks. Data for $\rho^{\prime}=1, \sigma^{\prime}=5$ (red circles); $\rho^{\prime}=3, \sigma^{\prime}=5$ (blue triangles); $\rho^{\prime}=4, \sigma^{\prime}=0.1$ (blue squares); and $\rho^{\prime}=8, \sigma^{\prime}=1$ (blue stars). Solid and broken lines correspond to Eq. (28).

Based on this correspondence, an equivalent Rayleigh number $\mathrm{Ra}_{\mathrm{eq}}$ is defined by taking the projection of the value of $\mathrm{Nu}$ in the fractured porous medium on the curve of the homogeneous medium as illustrated in Fig. 6. $\mathrm{Ra}_{\mathrm{eq}}$ is the value of $\mathrm{Ra}$ in a HPM which releases the same heat as a FPM.

$\mathrm{Ra}_{\text {eq }}$ is plotted as a function of $\mathrm{Ra}_{\mathrm{eff}}$ (see Sec. IVC) in Fig. 16, which shows that these two dimensionless quantities are linearly related.

Only four different networks are presented here for the sake of clarity, but computations were made on 48 different networks $\left(1 \leqslant \rho^{\prime} \leqslant 10\right.$ and $\left.0.1 \leqslant \sigma^{\prime} \leqslant 10\right)$ and the mean regression coefficient is equal to 0.998 .

Since a linear relationship relates $\mathrm{Ra}_{\mathrm{eq}}$ and $\mathrm{Ra} \mathrm{eff}_{\mathrm{ef}}, \mathrm{Ra} \mathrm{eq}_{\mathrm{eq}}$ depends only on the fracture network and on $\mathrm{Ra}_{m}$. This means that the heat released by a known fractured system could be possibly deduced from a homogeneous approach. The linear relationship can be analyzed further:

$$
\mathrm{Ra}_{\mathrm{eq}}=a \mathrm{Ra}_{\mathrm{eff}}+B,
$$

where $a$ and $B$ are fitted for each individual network. These coefficients are displayed in Fig. 17(a) for all the networks. They are seen to be correlated and aligned along the curve $a=1-B / 100$, with a regression coefficient of $r^{2}=0.991$ for nonpercolating networks and $r^{2}=0.881$ for percolating networks. Thus, Fig. 17(a) suggests the approximate relation

$$
\left(\mathrm{Ra}_{\mathrm{eq}}-100\right)=a\left(\mathrm{Ra}_{\mathrm{eff}}-100\right),
$$

where $a$ depends on the network realization. In particular, Eq. (29) is satisfied when $\mathrm{Ra}_{\mathrm{eq}}=\mathrm{Ra}_{\mathrm{eff}}=100$. As shown in Fig. 16, this solution is approximately verified for nonpercolating and some percolating networks. This value is very close to $\mathrm{Ra} \simeq 100.5$, for which two- and three-dimensional convections release the same energy (Fig. 5). Hence, it is not surprising that this solution is verified for networks inducing weak deformations of the flow patterns, such as nonpercolating networks and fracture networks with weak transmissivities.

Figures 17(b) and 17(c) display $a$ as a function of $\rho^{\prime}$ and $\sigma^{\prime}$, respectively; $a$ does not seem to be correlated to $\rho^{\prime}$ and $\sigma^{\prime}$, except for very weak transmissivities $\left(\sigma^{\prime} \ll 1\right)$, where $a$ is close to 1 , i.e., $\mathrm{Ra}_{\text {eff }} \approx \mathrm{Ra}_{\mathrm{eq}}$, which is in accordance with previous results (Sec. IV C). 

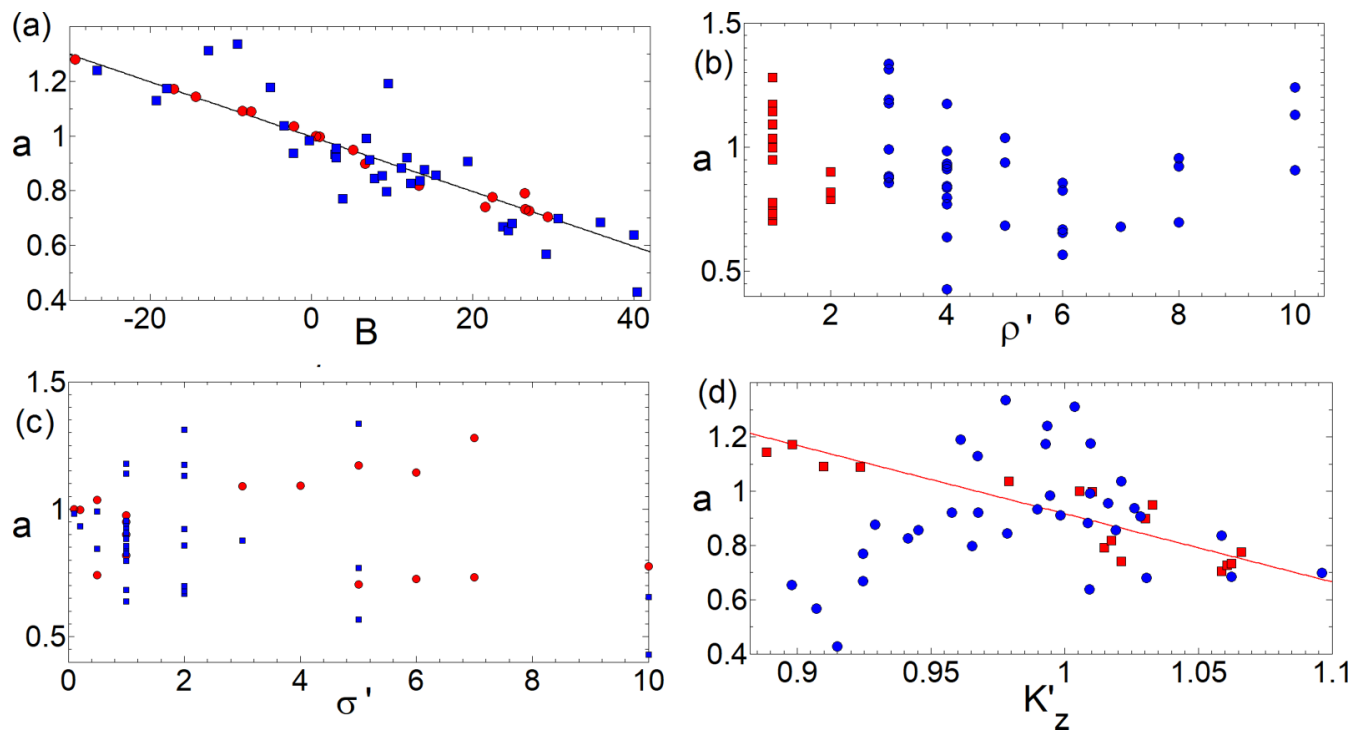

FIG. 17. The linear relation (29): $a$ as a function of (a) $B$, (b) $\rho^{\prime}$, (c) $b^{\prime}$, and (d) $K_{z}^{\prime}=2 K_{z} /\left(K_{x}+K_{y}\right.$ ). The solid line in (a) is $a=1-b / 100$ and that in (d) is $a=3.4-2.5 K_{z}^{\prime}$. Red dots and blue squares represent nonpercolating and percolating networks, respectively.

Another parameter is analyzed in order to check anisotropic effects:

$$
K_{z}^{\prime}=\frac{2 K_{\mathrm{eff}, z}}{K_{\mathrm{eff}, x}+K_{\mathrm{eff}, y}}
$$

where $K_{\text {eff, } x}, K_{\text {eff, } y}$, and $K_{\text {eff, } z}$ are the effective permeabilities calculated by imposing a pressure drop along the $x, y$, and $z$ axes, respectively. Figure 17(d) shows $a$ as a function of $K_{z}^{\prime}$. Some correlation between $a$ and $K_{z}^{\prime}$ can be observed for nonpercolating networks, namely, $a>1$ for the networks with a slight horizontal anisotropy $\left(K_{z}^{\prime}<1\right)$ and $a<1$ for the networks with a slight vertical anisotropy $\left(K_{z}^{\prime}>1\right)$. According to Eq. (29), for $\mathrm{Ra}_{\mathrm{eff}}<100, \mathrm{Ra}_{\mathrm{eq}}<\mathrm{Ra}_{\text {eff }}$ when $a>1$, where the effective medium approach underestimates the output thermal flux; these networks induce two-dimensional convection patterns. Moreover, $\mathrm{Ra}_{\mathrm{eq}}>\mathrm{Ra}_{\mathrm{eff}}$ when $a<1$, where the effective medium approach overestimates the output flux; the flow pattern is three dimensional. Therefore, it seems that nonpercolating networks with a slight horizontal anisotropy of the flow favor two-dimensional convection while nonpercolating networks with a slight vertical anisotropy of the flow promote three-dimensional convection. For percolating networks, no correlation between $K_{z}^{\prime}$ and $a$ was found.

To conclude, the relationship between $\mathrm{Ra}_{\text {eff }}$ and $\mathrm{Ra}_{\mathrm{eq}}$ makes possible the modeling of the heat transfer in a fractured reservoir by a homogeneous approach. Unfortunately, no way to predict the value of $\mathrm{Ra}_{\mathrm{eq}}$ for a particular FPM could be derived from the presented simulations.

\section{CONCLUSION AND PERSPECTIVES}

The influence of the parameters characterizing a fracture network, embedded in a permeable fully saturated porous matrix, has been investigated by a direct approach which takes into account the real geometry of the fracture network and the distribution of the physical properties. This study is limited to closed-top cubic boxes, but other boundary conditions can be easily included in the numerical model.
The results provide a quantitative estimate of the heat released by the medium, when the density of fractures and their transmissivity are varied. More precisely, the output flux increases linearly with the fracture density, in the investigated ranges of Rayleigh number, density, and fracture transmissivity. The influence of transmissivity on the output flux shows the importance of the network percolation status. However, the percolation status of a fracture network when flow is governed by a pressure drop consists of a cluster of fractures, forming a connected path joining two opposite boundaries of the domain [29]. Since in this study the flow is governed by thermal convection, the percolation status should refer to a connected set of fractures following the whole trajectory of the convection cell.

The comparison of the heat transfer in a fractured medium and in a homogeneous medium with the same effective properties shows that predictions based on the effective permeability are not satisfactory in a limited range of densities. The heat transfer occurring in a fractured porous medium using a homogeneous porous approach, considering the same initial conditions and the effective permeability deduced from Ref. [29], is reasonably predicted (within 20\%) for $\rho^{\prime} \leqslant 2$ and $\rho^{\prime} \geqslant 4$. These $20 \%$ represent the fluctuations of the Nusselt number for various networks with the same $\rho^{\prime}$, related to the particular geometry of each network. However, on a caseby-case basis, the homogeneous porous approach gives better results when $\rho^{\prime}$ is very small or very dense. The results show that for $\rho^{\prime} \leqslant 2$ and $\rho^{\prime} \geqslant 8$ the effective approach predicts $\mathrm{Nu}$ within $10 \%$.

This study highlights the existence of an equivalent Rayleigh number $\mathrm{Ra}_{\mathrm{eq}}$ in a fractured porous medium, which is linearly related to the effective Rayleigh number $\mathrm{Ra}_{\mathrm{eff}}$ in a homogeneous porous medium and by extension to the Rayleigh number in the porous matrix containing the fracture network. This linearity implies that the heat release from a fractured medium could be known by means of a homogeneous approach. However, the way to estimate the coefficient linking $\mathrm{Ra}_{\mathrm{eq}}$ and $\mathrm{Ra}_{m}$ for a particular fractured network is not elucidated in this study. 
Finally, this study could be extended in many ways. Various boundary conditions could be chosen in order to schematize various geological situations. Presently, the influence of an open-top condition and the influence of the anisotropy of the fracture network and non-Boussinesq effects when the fluid viscosity and thermal expansivity vary with temperature are being studied.

\section{ACKNOWLEDGMENTS}

The authors would like to thank Electricité de France for funding the Ph.D. work of C.M. Part of this work was performed when P.M.A. was supported at the Mechanical Engineering Department, Technion, Haifa, Israel, by a fellowship of the Lady Davis Foundation.
[1] D. A. Neld and A. Bejan, Convection in Porous Media, 3rd ed. (Springer, Berlin, 2006).

[2] C. W. Horton and F. T. Rogers, Jr., Convection currents in a porous medium, J. Appl. Phys. 16, 367 (1945).

[3] E. R. Lapwood, Convection of a fluid in a porous medium, Proc. Cambridge Philos. Soc. 44, 508 (1948).

[4] D. Nield, Onset of thermohaline convection in a porous medium, Water Resour. Res. 4, 553 (1968).

[5] H. L. Morrison, Preliminary measurements relative to the onset of thermal convection currents in unconsolidated sands, J. Appl. Phys. 18, 849 (1947).

[6] H. L. Morrison, F. T. Rogers, Jr., and C. W. Horton, Convection currents in porous media: II. Observation of conditions at onset of convection, J. Appl. Phys. 20, 1027 (1949).

[7] R. Wooding, Steady state free thermal convection of liquid in a saturated permeable medium, J. Fluid Mech. 2, 273 (1957).

[8] J. W. Elder, Transient convection in a porous medium, J. Fluid Mech. 27, 609 (1966).

[9] M. A. Combarnous and P. Bia, Combined free and forced convection in porous media, Soc. Pet. Eng. J. 11, 399 (1971).

[10] M. Combarnous and S. Bories, Modélisation de la convection naturelle au sein d'une couche poreuse horizontale a l'aide d'un coefficient de transfert solide-fluide, Int. J. Heat Mass Transf. 17, 505 (1974).

[11] J. M. Straus, Large amplitude convection in porous media, J. Fluid Mech. 64, 51 (1974).

[12] G. Schubert and J. M. Straus, Three-dimensional and multicellular steady and unsteady convection in fluid-saturated porous media at high Rayleigh numbers, J. Fluid Mech. 94, 25 (1979).

[13] G. F. Pinder, State-of-the-art review of geothermal reservoir modeling, Lawrence Berkeley Laboratory, University of California, Report No. LBL-9093, GSRMP-5, UC-66a, 1979 (unpublished).

[14] G. I. Barenblatt and I. P. Zheltov, Basic concepts in the theory of seepage of homogeneous liquids in fissured rocks, J. Appl. Math. Mech. 24, 1286 (1960).

[15] G. I. Barenblatt and Yu. P. Zheltov, Fundamental equations of filtration of homogeneous liquids in fissured rocks, Dokl. Akad. Nauk SSSR 132, 545 (1960) [Sov. Phys. Dokl. 5, 522 (1960)].

[16] K. Pruess, A practical method for modeling fluid and heat flow in fractured porous media, Soc. Pet. Eng. J. 25, 14 (1985).

[17] K. H. Williamson, Reservoir simulation of The Geysers geothermal field, in Proceedings, Fifteenth Workshop on Geothermal Reservoir Engineering, H. J. Ramey, Jr., P. Kruger, F. G. Miller, R. N. Horne, W. E. Brigham, J. W. Cook (eds.), Stanford University, Stanford, CA, Report No. SGP-TR-130, 1990, p.113123. (unpublished).
[18] S. Nakanishi and N. Iwai, Reservoir simulation study of the Onikobe geothermal field, Japan, in Proceedings of the World Geothermal Congress, 2000, edited by E. Iglesias, D. Blackwell, T. Hunt, J. Lund, S. Tamanyu, and K. Kimbara (International Geothermal Association, Kyushu-Tohoku, Japan, 2000), pp. 2159-2164.

[19] M. J. O'Sullivan, K. Pruess, and M. J. Lippmann, State of the art of geothermal reservoir simulation, Geothermics 30, 395 (2001).

[20] G. A. Simms and G. Garven, Thermal convection in faulted extensional sedimentary basins: Theoretical results from finiteelement modeling, Geofluids 4, 109 (2004).

[21] A. Bataillé, P. Genthon, M. Rabinowicz, and B. Fritz, Modeling the coupling between free and forced convection in a vertical permeable slot: Implications for the heat production of an enhanced geothermal system, Geothermics 35, 654 (2006).

[22] F. Magri, T. Akar, U. Gemici, and A. Pekdeger, Deep geothermal groundwater flow in the Seferihisar-Balçova area, Turkey: Results from transient numerical simulations of coupled fluid flow and heat transport processes, Geofluids 10, 388 (2010).

[23] M. Onur, N. Aksoy, U. Serpen, and A. Satman, Analysis of pressure transient tests in Balcova-Narlidere geothermal field, Turk. J. Oil Gas 8, 20 (2002).

[24] V. Harcouet-Menou, L. Guillou-Frottier, A. Bonneville, P. M. Adler, and V. Mourzenko, Hydrothermal convection in and around mineralized fault zones: Insights from two- and threedimensional numerical modeling applied to the Ashanti belt, Ghana, Geofluids 9, 116 (2009).

[25] M. G. Blöcher, M. Cacace, G. Lewerenz, and G. Zimmermann, Three dimensional modelling of fractured and faulted reservoirs: Framework and implementation, Chem. Erde 70, 145 (2010).

[26] M. Cacace, M. G. Blöcher, N. Watanabe, I. Moeck, N. Börsing, M. Scheck-wenderoth, O. Kolditz, and E. Huenges, Modelling of fractured carbonate reservoirs: Outline of a novel technique via a case study from the Molasse Basin, southern Bavaria, Germany, Environ. Earth Sci. 70, 3585 (2013).

[27] Y. Cherubini, M. Cacace, M. G. Blöcher, and M. ScheckWenderoth, Impact of single inclined faults on the fluid flow and heat transport: Results from 3-D finite element simulations, Environ. Earth Sci. 70, 3603 (2013).

[28] O. Kolditz et al., OpenGeoSys: An open-source initiative for numerical simulation of thermo-hydro-mechanical/chemical (THM/C) processes in porous media, Environ. Earth Sci. 67, 589 (2012).

[29] P. M. Adler, J.-F. Thovert, and V. V. Mourzenko, Fractured Porous Media (Oxford University Press, Oxford, 2012).

[30] I. Bogdanov, V. V. Mourzenko, J.-F. Thovert, and P. M. Adler, Effective permeability of fractured porous media in steady state flow, Water Resour. Res. 39, 1023 (2003). 
[31] I. Bogdanov, V. V. Mourzenko, J.-F. Thovert, and P. M. Adler, Two-phase flow through fractured porous media, Phys. Rev. E 68, 026703 (2003).

[32] P. Cheng, Heat transfer in geothermal systems, Adv. Heat Transfer 14, 1 (1978).

[33] E. A. Sudicky and R. G. McLaren, The Laplace transform Galerkin technique for large-scale simulation of mass transport in discretely fractured porous formations, Water Resour. Res. 28, 499 (1992).

[34] S. G. Shikaze, E. A. Sudicky, and F. W. Schwartz, Densitydependent solute transport in discretely-fractured geologic media: Is prediction possible?, J. Contam. Hydrol. 34, 273 (1998).

[35] O. Huseby, J.-F. Thovert, and P. M. Adler, Geometry and topology of fracture systems, J. Phys. A: Math. Theor. 30, 1415 (1997).

[36] N. Koudina, G. R. Gonzalez, and J.-F. Thovert, Permeability of three-dimensional fracture networks, Phys. Rev. E 57, 4466 (1998).

[37] K. Vujević, T. Graf, C. T. Simmons, and A. D. Werner, Impact of fracture network geometry on free convective flow patterns, Adv. Water Resour. 71, 65 (2014).

[38] D. Kuzmin and S. Turek, High-resolution FEM-TVD schemes based on a fully multidimensional flux limiter, J. Comput. Phys. 198, 131 (2004).
[39] J. M. Straus and G. Schubert, Three-dimensional convection in a cubic box of fluid-saturated porous material, J. Fluid Mech. 91, 155 (1979).

[40] S. Kimura, G. Schubert, and J. M. Straus, Time-dependent convection in a fluid-saturated porous cube heated from below, J. Fluid Mech. 207, 153 (1989).

[41] A. S. M. Cherkaoui and W. S. D. Wilcock, Characteristics of high Rayleigh number two-dimensional convection in an open-top porous layer heated from below, J. Fluid Mech. 394, 241 (1999).

[42] A. Zebib and D. R. Kassoy, Three-dimensional natural convection motion in a confined porous medium, Phys. Fluids 21, 1 (1977).

[43] V. V. Mourzenko, J.-F. Thovert, and P. M. Adler, Percolation of three-dimensional fracture networks with power-law size distribution, Phys. Rev. E 72, 036103 (2005).

[44] J. C. S. Long, J. S. Remer, C. R. Wilson, and P. A. Witherspoon, Porous media equivalents for networks of discontinuous fractures, Water Resour. Res. 18, 645 (1982).

[45] V. V. Mourzenko, J.-F. Thovert, and P. M. Adler, Permeability of isotropic and anisotropic fracture networks, from the percolation threshold to very large densities, Phys. Rev. E 84, 036307 (2011).

[46] M. E. Fischer, The theory of critical point singularities, in Critical Phenomena, Proceedings of the International School of Physics "Enrico Fermi” Course LI, Varenna, edited by M. S. Green (Academic Press, New York, 1971). 